Research Article

Open Access

Special Issue: Model Uncertainty and Robust Finance

Daniel Lacker*

\title{
Law invariant risk measures and information divergences
}

https://doi.org/10.1515/demo-2018-0014

Received April 17, 2018; accepted October 2, 2018

Abstract: A one-to-one correspondence is drawn between law invariant risk measures and divergences, which we define as functionals of pairs of probability measures on arbitrary standard Borel spaces satisfying a few natural properties. Divergences include many classical information divergence measures, such as relative entropy and convex $f$-divergences. Several properties of divergence and their duality with law invariant risk measures are characterized, such as joint semicontinuity and convexity, and we notably relate their chain rules or additivity properties with certain notions of time consistency for dynamic law risk measures known as acceptance and rejection consistency. The examples of shortfall risk measures and optimized certainty equivalents are discussed in detail.

Keywords: Risk measures, law invariance, information divergence, time consistency

MSC: 91B30, 94A17

\section{Introduction}

This paper deepens the analysis of law invariant convex risk measures and their duality with functionals of probability measures. The main contributions are (1) to show how the usual convex conjugate or penalty function can, in the law-invariant case, be viewed as a divergence-like functional of not one but two probability measures, and (2) to characterize several natural properties of these functionals, pertaining to convexity, continuity, and time consistency, in terms of properties of the corresponding risk measure and its acceptance set.

Throughout the paper, a nonatomic standard Borel space $(\Omega, \mathcal{F}, P)$ is fixed, and a risk measure is defined to be a convex functional $\rho: L^{\infty}:=L^{\infty}(\Omega, \mathcal{F}, P) \rightarrow \mathbb{R}$ satisfying:

1. Monotonicity: If $X, Y \in L^{\infty}$ and $X \leq Y$ a.s. then $\rho(X) \leq \rho(Y)$.

2. Cash additivity: If $X \in L^{\infty}$ and $c \in \mathbb{R}$ then $\rho(X+c)=\rho(X)+c$.

3. Normalization: $\rho(0)=0$.

The functional $X \mapsto \rho(-X)$ is more traditionally called a normalized convex risk measure; some authors use the term acceptability measure [34] for what we have chosen to call a risk measure. A risk measure $\rho$ is law invariant if $\rho(X)=\rho(Y)$ whenever $X$ and $Y$ have the same law. Three standard examples will guide us throughout the paper: The first is the well known entropic risk measure $\rho(X)=\eta^{-1} \log \mathbb{E}\left[e^{\eta X}\right], \eta>0$. Second, given a nondecreasing convex function $\ell: \mathbb{R} \rightarrow[0, \infty)$ with $\ell(0)=1$, the corresponding shortfall risk measure (introduced by Föllmer and Schied in [17]) is

$$
\rho(X)=\inf \{c \in \mathbb{R}: \mathbb{E}[\ell(X-c)] \leq 1\} .
$$

${ }^{\star}$ Corresponding Author: Daniel Lacker: Columbia University, Department of Industrial Engineering \& Operations Research,

E-mail: daniel.lacker@columbia.edu

Әopen Access. (c) BY-NC-ND $\odot 2018$ Daniel Lacker, published by De Gruyter. This work is licensed under the Creative Commons AttributionNonCommercial-NoDerivs 4.0 License. 
Lastly, given a nondecreasing convex function $\phi: \mathbb{R} \rightarrow \mathbb{R}$ with $\phi^{\star}(1)=\sup _{x \in \mathbb{R}}(x-\phi(x))=0$, the corresponding optimized certainty equivalent (introduced by Ben-Tal and Teboulle in $[7,8]$ ) is

$$
\rho(X):=\inf _{m \in \mathbb{R}}(\mathbb{E}[\phi(m+X)]-m) .
$$

We construct divergences as follows: Fix a law invariant risk measure $\rho$. Given a Polish space $E$, let $\mathcal{P}(E)$ denote the set of Borel probability measures on $E$. For any Polish space (or any standard Borel space) $E$ and any $\mu \in \mathcal{P}(E)$, we may define a new law invariant risk measure $\rho_{\mu}: L^{\infty}(E, \mu) \rightarrow \mathbb{R}$ by $\rho_{\mu}(f):=\rho(f(X))$, where $X$ is any $E$-valued random variable on $\Omega$ with law $P \circ X^{-1}=\mu$. Indeed, such an $X$ exists because $\Omega$ is nonatomic, and this definition is independent of the choice $X$ thanks to law invariance. This family of risk measures satisfies a consistency property, namely

$$
\rho_{\mu}(f)=\rho_{\nu}(g) \text {, whenever } \mu \circ f^{-1}=\nu \circ g^{-1} .
$$

Let $\alpha(\cdot \mid \mu)$ denote the minimal penalty function associated to $\rho_{\mu}$, i.e., the restriction to $\mathcal{P}(E)$ of the convex conjugate of $\rho_{\mu}$ :

$$
\alpha(v \mid \mu)=\sup _{f \in L^{\infty}(E, \mu)}\left(\int_{E} f d v-\rho_{\mu}(f)\right)=\sup \left\{\int_{E} f d v: f \in L^{\infty}(E, \mu), \rho_{\mu}(f) \leq 0\right\} .
$$

We call $\alpha$ the divergence induced by $\rho$. In summary, the functional $\alpha(\cdot \mid \cdot)$ is defined for pairs of probability measures on any Polish space (or standard Borel space), much like the classical relative entropy and other information divergences, such as the $f$-divergence [31, 32]. Indeed, when $\rho$ is the entropic risk measure, $\alpha$ is nothing but the usual relative entropy (also known as the Kullback-Leibler divergence),

$$
H(v \mid \mu)=\int \log \left(\frac{d v}{d \mu}\right) d v \text { for } v \ll \mu, \quad \infty \text { otherwise. }
$$

As a first key result, we show that the map from $\rho$ to $\alpha$ can be inverted: Consider a $[0, \infty]$-valued function $\alpha=\alpha(v \mid \mu)$, defined for pairs of probability measures $(\nu, \mu) \in \mathcal{P}(E)^{2}$ for any Polish space $E$, such that for each Polish space $E$ and each $\mu \in \mathcal{P}(E)$ we have the following properties:

1. $\alpha(\mu \mid \mu)=0$.

2. $\alpha(v \mid \mu)=\infty$ if $v \in \mathcal{P}(E)$ is not absolutely continuous with respect to $\mu$.

3. The map $v \mapsto \alpha(v \mid \mu)$ is convex and lower semicontinuous with respect to total variation.

4. $\alpha(v K \mid \mu K) \leq \alpha(v \mid \mu)$ for every $v \in \mathcal{P}(E)$ and every kernel $K$ from $E$ to another Polish space $F$, where $\mu K(d y):=\int_{E} \mu(d x) K(x, d y) \in \mathcal{P}(F)$.

We call such a functional a divergence, and we show that to any divergence there corresponds a unique law invariant risk measure defined on the original space $(\Omega, \mathcal{F}, P)$; we prove this by showing the definitions

$$
\rho_{\mu}(f):=\sup _{v \in \mathcal{P}(E)}\left(\int_{E} f d v-\alpha(v \mid \mu)\right),
$$

to be consistent in the sense of (1.1), where $E$ is a Polish space, $f \in B(E)$, and $\mu=P \circ X^{-1}$ for some $X$ : $\Omega \rightarrow E$. The property (4) corresponds exactly to the consistency property (1.1) and is known as the data processing inequality in information theory, at least when $\alpha$ is the usual relative entropy. It is worth noting that, while the terminology divergence is natural here for many reasons, our definition excludes many natural examples, such as non-convex $f$-divergences (e.g., the Hellinger distance) and all norms or metrics on the space of probability measures, as the latter would violate property (2) above.

Our work first catalogs several interesting properties of these divergence functionals, characterizing when they are jointly lower semicontinuous or jointly convex in $(\nu, \mu)$. We show that joint lower semicontinuity with respect to the topology of weak convergence turns out to be equivalent to Lebesgue continuity of 
the risk measure $\rho$ (i.e., continuity of $\rho$ with respect to bounded pointwise convergence). On the other hand, we show that joint convexity of a divergence is equivalent to concavity of $\rho$ on the level of distributions, a property studied in some detail by Acciaio and Svindland [2] and which holds for every optimized certainty equivalent.

A substantial focus of the paper, undertaken in Section 5, is on the characterization of properties of divergences related to the well known chain rule for relative entropy, which reads

$$
H\left(v(d x) K_{x}^{v}(d y) \mid \mu(d x) K_{x}^{\mu}(d y)\right)=H(v \mid \mu)+\int v(d x) H\left(K_{x}^{v} \mid K_{x}^{\mu}\right),
$$

and holds for all (disintegrated) probability measures $\mu(d x) K_{x}^{\mu}(d y)$ and $v(d x) K_{x}^{v}(d y)$ on the product of two Polish spaces. More generally, we say a divergence $\alpha$ is superadditive if

$$
\alpha\left(v(d x) K_{x}^{v}(d y) \mid \mu(d x) K_{x}^{\mu}(d y)\right) \geq \alpha(v \mid \mu)+\int v(d x) \alpha\left(K_{x}^{v} \mid K_{x}^{\mu}\right) .
$$

and we say $\alpha$ is subadditive if the reverse inequality holds. The original motivation for this investigation comes from an ongoing investigation into properties of concentration inequalities of the form $\rho(\lambda X) \leq \gamma(\lambda)$ for all $\lambda \geq 0$, where $\gamma:[0, \infty) \rightarrow[0, \infty]$. These inequalities are well-understood when $\rho$ is the entropic risk measure, and in a follow-up paper [30] we study the general case in connection with liquidity risk. Our results on superadditivity underlie the study of tensorization of these inequalities [30, Section 5] as well as the recent limit theorems of $[14,29]$, which generalize the classical large deviation results on empirical measures of i.i.d. samples and Markov chains.

It turns out that the dual form of superadditivity (1.2) is a so-called time-consistency property of the corresponding risk measure $\rho$, which we describe by building on a construction of Weber [38]: Define a functional $\tilde{\rho}$ on $\mathcal{P}(\mathbb{R})$ by $\tilde{\rho}\left(P \circ X^{-1}\right)=\rho(X)$, which is of course well defined thanks to law invariance. For any $\sigma$-field $\mathcal{G} \subset \mathcal{F}$ in $\Omega$ and any $X \in L^{\infty}$, consider the $\mathcal{G}$-measurable random variable

$$
\rho(X \mid \mathcal{G})(\omega):=\tilde{\rho}(P(X \in \cdot \mid \mathcal{G})(\omega)),
$$

where $P(X \in \cdot \mid \mathcal{G})$ denotes a regular conditional law of $X$ given $\mathcal{G}$. We say $\rho$ is acceptance consistent if $\rho(X) \leq \rho(\rho(X \mid \mathcal{G}))$ for every $X \in L^{\infty}$ and any $\sigma$-field $\mathcal{G} \subset \mathcal{F}$. If the reverse inequality holds, we say $\rho$ is $r e$ jection consistent. If $\rho$ is both acceptance and rejection consistent, we say it is time consistent. We show that acceptance consistency of $\rho$ is essentially equivalent to the superadditivity of the induced divergence $\alpha$, and we provide an additional characterization in terms of a property of the measure acceptance set

$$
\mathcal{A}:=\left\{P \circ X^{-1}: X \in L^{\infty}, \rho(X) \leq 0\right\} \subset \mathcal{P}(\mathbb{R}) .
$$

These various characterizations are put to use to identify which shortfall risk measures and optimized certainty equivalents are acceptance consistent. The results of Kupper and Schachermayer [27] imply that the entropic risk measure is essentially the only time consistent risk measure, and as a corollary we find that the relative entropy is the only divergence (up to a scalar multiple) satisfying the chain rule. ${ }^{1}$ Ultimately, we find that not many law invariant risk measures are acceptance consistent (or rejection consistent) other than the entropic one, or modest perturbations thereof. In other words, not many divergences beyond relative entropy are superadditive. Although our results are somewhat negative in this sense, the divergences we study are interesting in their own right, and they appear to be useful tools in the study of law invariant risk measures. Moreover, we find some value in understanding the limitations of our divergences in the applications to concentration of measure and large deviations discussed in the previous paragraph.

We also briefly revisit the related results of Weber [38]. Say that $\rho$ is weakly acceptance consistent if $\rho(X) \leq$ 0 whenever $\rho(X \mid \mathcal{G}) \leq 0$ a.s., for $X \in L^{\infty}$ and $\sigma$-fields $\mathcal{G} \subset \mathcal{F}$. Weber showed that this is essentially equivalent to

1 We make no attempt to reconcile our characterization of relative entropy with the many already present in the literature (see the survey of Csiszár [11]), but we can at least say with confidence that the techniques by which we obtained it are new, notably avoiding functional equations. 
the convexity of the measure acceptance set $\mathcal{A}$. We show that weak acceptance consistency is also equivalent to an inequality weaker than superadditivity:

$$
\alpha\left(v(d x) K_{x}^{v}(d y) \mid \mu(d x) K_{x}^{\mu}(d y)\right) \geq \int v(d x) \alpha\left(K_{x}^{v} \mid K_{x}^{\mu}\right) .
$$

Convex risk measures first appeared in [17, 19, 22], extending the class of coherent risk measures introduced in the seminal paper of Artzner et al. [4] (see also [12]). Time consistency properties of dynamic risk measures have by now been studied thoroughly [10, 13, 16, 20, 33, 37]. The nice survey of Acciaio and Penner [1] will be a useful reference, although we will mostly work with the type of dynamic law invariant risk measures constructed by Weber in [38]. With this rich literature in mind, the most novel of our results on time consistency is the characterization of acceptance consistency in terms of a property of the measure acceptance set, which nicely complements Weber's result on weak acceptance consistency. Our characterization in terms of superadditivity could likely be deduced from results in the recent [5], which appeared after the first version of our paper. We retain full proofs for the sake of completeness and because our setting is somewhat different.

The paper is organized as follows. Section 2 reviews the basic definitions and duality results of law invariant risk measures before introducing divergences and studying their first properties. The characterization of divergences in terms of law invariant risk measures is given by Proposition 2.6 and Theorem 2.7. The short Sections 3 and 4 provide the natural dual characterizations of joint lower semicontinuity and joint convexity of divergences. Section 5 then turns to time consistency and superadditivity: The main Theorem 5.5 characterizes time consistency properties of a law invariant risk measure in terms of both the induced divergence and the measure acceptance set. Section 6 studies additional information-theoretic properties of divergences, and finally Section 7 applies the theory to the examples of shortfall risk measures and optimized certainty equivalents.

\section{Risk measures and divergences}

First, let us fix some notation. Throughout the paper, $(\Omega, \mathcal{F}, P)$ is a fixed probability space, which we assume is a nonatomic standard Borel space. Abbreviate $L^{p}=L^{p}(\Omega, \mathcal{F}, P)$ as usual for the set of (equivalence classes of) $p$-integrable real-valued measurable functions on $\Omega$. Let $\mathcal{P}(\Omega)$ denote the set of probability measures on $(\Omega, \mathcal{F})$, and let $\mathcal{P}_{P}(\Omega)$ denote the subset consisting of those measures which are absolutely continuous with respect to $P$. As stated in the introduction, a risk measure to us is a convex nondecreasing (with respect to a.s. order) functional $\rho: L^{\infty} \rightarrow \mathbb{R}$ satisfying $\rho(0)=0$ and $\rho(X+c)=\rho(X)+c$ for all $X \in L^{\infty}, c \in \mathbb{R}$. Note again that this is somewhat different from the standard definition, in which $\rho$ is instead nonincreasing [18]. Law-invariant risk measures possess some nice additional structure, highlighted in particular by the results of [23] and [15], though we will not need the latter.

Theorem 2.1 (Theorem 2.1 of [23]). Every law-invariant risk measure $\rho$ satisfies the Fatou property, which means that whenever $X_{n} \in L^{\infty}$ are uniformly bounded and converge a.s. to $X \in L^{\infty}$, then $\rho(X) \leq$ $\liminf _{n \rightarrow \infty} \rho\left(X_{n}\right)$.

Let us recall a classical duality result, but note that the details of the presentation are somewhat unusual: We say a function $\alpha: \mathcal{P}_{P}(\Omega) \rightarrow[0, \infty]$ is a penalty function for $\rho$ if it holds that

$$
\rho(X)=\sup _{Q \in \mathcal{P}_{P}(\Omega)}\left(\mathbb{E}^{Q}[X]-\alpha(Q)\right) .
$$

(Note that the supremum involves only countably additive measures, and we will make no mention of finite additivity.) Here $\mathbb{E}^{Q}$ denotes expectation with respect to the probability $Q$. Expectation under the reference measure $P$ is simply denoted $\mathbb{E}$, and integrals on spaces other than $\Omega$ are written in a more explicit measuretheoretic notation. 
Theorem 2.2 (Theorem 4.33 of [18]). Suppose $\rho$ is a law invariant risk measure. Then the function $\alpha: \mathcal{P}_{P}(\Omega) \rightarrow$ $[0, \infty]$ defined by

$$
\alpha(Q):=\sup \left\{\mathbb{E}^{Q}[X]: X \in L^{\infty}, \rho(X) \leq 0\right\}=\sup _{X \in L^{\infty}}\left\{\mathbb{E}^{Q}[X]-\rho(X)\right\}
$$

is a penalty function for $\rho$. In fact, it is the minimal penalty function, in the sense that any other penalty function $\alpha^{\prime}$ for $\rho$ satisfies $\alpha \leq \alpha^{\prime}$.

Note that the dual representation (2.2) implies that the minimal penalty function is convex and lower semicontinuous with respect to the total variation topology, as well as the weaker topology $\sigma\left(\mathcal{P}_{P}(\Omega), L^{\infty}\right)$. $^{2}$ There is an alternative dual representation more specific to law invariant risk measures, due to Kusuoka [28] and extended in $[21,23]$, but we will make no use of this.

Remark 2.3. Note that we may afford to be lazy about the fact that $\rho$ is to be evaluated at equivalence classes, i.e. elements of $L^{\infty}$, as opposed to specific measurable functions. For a risk measure $\rho$, we may define $\rho(X):=$ $\rho([X])$ in the obvious way for a measurable function $X: \Omega \rightarrow \mathbb{R}$ by finding the equivalence class $[X] \in L^{\infty}$ to which $X$ belongs. With this in mind, we may then define $\alpha(Q):=\infty$ for $Q \in \mathcal{P}(\Omega)$ which are not absolutely continuous with respect to $P$, and then the dual formula (2.1) may be re-written

$$
\rho(X)=\sup _{Q \in \mathcal{P}(\Omega)}\left(\mathbb{E}^{Q}[X]-\alpha(Q)\right),
$$

for bounded measurable functions $X: \Omega \rightarrow \mathbb{R}$.

As with many properties of convex risk measures, law invariance may be alternatively characterized by a property of the minimal penalty function, and this will be a building block for a more general discussion in the next section. This characterization appears to be new, although a very similar result appeared in [35, Proposition 2], and see also [23, Lemma A.4].

Proposition 2.4. Suppose a risk measure $\rho$ on $L^{\infty}$ has the Fatou property (see Theorem 2.1). Then $\rho$ is lawinvariant if and only if it has a penalty function $\alpha$ satisfying $\alpha\left(Q \circ T^{-1}\right) \leq \alpha(Q)$ for every $Q \in \mathcal{P}_{P}(\Omega)$ and for every measurable $T: \Omega \rightarrow \Omega$ satisfying $P \circ T^{-1}=P$.

Proof. First, assume $\rho$ is law invariant, and let $\alpha$ be its minimal penalty function provided by Theorem 2.2. Let $T: \Omega \rightarrow \Omega$ be a measurable map satisfying $P \circ T^{-1}=P$. Then $X \circ T$ and $X$ have the same law and thus $\rho(X)=\rho(X \circ T)$ for every $X \in L^{\infty}$. Hence

$$
\begin{aligned}
\alpha\left(Q \circ T^{-1}\right) & =\sup _{X \in L^{\infty}}\left(\mathbb{E}^{Q \circ T^{-1}}[X]-\rho(X)\right) \\
& =\sup _{X \in L^{\infty}}\left(\mathbb{E}^{Q}[X \circ T]-\rho(X \circ T)\right) \\
& \leq \sup _{X \in L^{\infty}}\left(\mathbb{E}^{Q}[X]-\rho(X)\right) \\
& =\alpha(Q) .
\end{aligned}
$$

2 As usual, when $F$ is a set of real-valued functions on a set $E$, the notation $\sigma(E, F)$ refers to the coarsest topology on $E$ rendering the elements of $F$ continuous. 
To prove the converse, fix $X, Y \in L^{\infty}$ with the same law. By [24, Corollary 6.11] (since the probability space is nonatomic) we may find a measurable map $T: \Omega \rightarrow \Omega$ such that $P \circ T^{-1}=P$ and $P(X=Y \circ T)=1$. Then

$$
\begin{aligned}
\rho(X) & =\sup _{Q \in \mathcal{P}_{P}(\Omega)}\left(\mathbb{E}^{Q}[X]-\alpha(Q)\right) \\
& =\sup _{Q \in \mathcal{P}_{P}(\Omega)}\left(\mathbb{E}^{Q \circ T^{-1}}[Y]-\alpha(Q)\right) \\
& \leq \sup _{Q \in \mathcal{P}_{P}(\Omega)}\left(\mathbb{E}^{Q \circ T^{-1}}[Y]-\alpha\left(Q \circ T^{-1}\right)\right) \\
& \leq \sup _{Q \in \mathcal{P}_{P}(\Omega)}\left(\mathbb{E}^{Q}[Y]-\alpha(Q)\right) \\
& =\rho(Y) .
\end{aligned}
$$

Reversing the roles of $X$ and $Y$ completes the proof.

Remark 2.5. From the proof of Proposition 2.4, it should be clear that the assumption that $\rho$ has the Fatou property is not needed. We state only this simpler form in order to avoid introducing additional terminology, and to avoid dwelling on details involving finitely additive measures.

\subsection{Divergences and their characterization}

Let us now exploit law invariance to construct a corresponding family of risk measures and what we refer to as divergences. Fix for the rest of this section a law-invariant risk measure $\rho$. Given a Polish space $E$, let $\mathcal{P}(E)$ denote the set of Borel probability measures on $E$. We write $v \ll \mu$ to mean $v$ is absolutely continuous with respect to $\mu$. Given any $\mu \in \mathcal{P}(E)$, write $\mathcal{P}_{\mu}(E):=\{v \in \mathcal{P}(E): v \ll \mu\}$. Define also $C(E), C_{b}(E)$, and $B(E)$ to be the sets of continuous, bounded continuous, and bounded measurable functions on $E$, respectively. The space $\mathcal{P}(E)$ is endowed with the $\sigma$-field generated by the maps $\mu \mapsto \mu(A)$, where $A \subset E$ is Borel; this equals the Borel $\sigma$-field generated by the topology of weak convergence, i.e., $\sigma\left(\mathcal{P}(E), C_{b}(E)\right.$ ).

Given a Polish space $E$ and $\mu \in \mathcal{P}(E)$, we may find (because $\Omega$ is nonatomic) a measurable function $X: \Omega \rightarrow E$ such that $P \circ X^{-1}=\mu$. We may then define a (law invariant) risk measure $\rho_{\mu}$ on $L^{\infty}(E, \mu)$ by

$$
\rho_{\mu}(f):=\rho(f(X)) .
$$

Note that by law-invariance this definition does not depend on the choice of $X$, as long as $P \circ X^{-1}=\mu$. We call $\left(\rho_{\mu}\right)_{\mu, E}$ the family of risk measures induced by $\rho$. This family of risk measures satisfies a consistency property, namely

$$
\rho_{\mu}(f)=\rho_{\nu}(g) \text {, whenever } \mu \circ f^{-1}=v \circ g^{-1} .
$$

In particular, for any measurable map $T$ from one Polish space $E$ to another $F$, we have $\rho_{\mu \circ T^{-1}}(f)=\rho_{\mu}(f \circ T)$, for $f \in L^{\infty}\left(F, \mu \circ T^{-1}\right)$. The same construction is valid when $E$ is any standard Borel space, but for simplicity we stick with Polish spaces.

The minimal penalty function of $\rho_{\mu}$ is denoted $\alpha(\cdot \mid \mu): \mathcal{P}_{\mu}(E) \rightarrow[0, \infty]$ and defined by

$$
\alpha(v \mid \mu):=\sup _{f \in B(E)}\left(\int_{E} f d v-\rho_{\mu}(f)\right)=\sup \left\{\int_{E} f d v: f \in L^{\infty}(E, \mu), \rho_{\mu}(f) \leq 0\right\} .
$$

Extend $\alpha(\cdot \mid \mu)$ to all of $\mathcal{P}(E)$ by setting $\alpha(v \mid \mu)=\infty$ whenever $\nu$ is not absolutely continuous with respect to $\mu$. Then, for $f \in B(E)$,

$$
\rho(f(X))=\rho_{\mu}(f)=\sup _{v \in \mathcal{P}(E)}\left(\int_{E} f d v-\alpha(v \mid \mu)\right), \text { if } P \circ X^{-1}=\mu,
$$


and it is easy to check that (2.4) remains valid for $v \in \mathcal{P}(E) \backslash \mathcal{P}_{\mu}(E)$. (As in Remark 2.3, let us not be overly careful about distinguishing between measurable functions and equivalence classes thereof.) We refer to $\alpha(\cdot \cdot \cdot)$ as the divergence induced by $\rho$. Note that $\alpha(\cdot \mid \cdot)$ is defined for pairs of probability measures on any Polish space. Additionally, $\alpha(\cdot \mid \mu)$ is always convex and lower semicontinuous with respect to total variation, and also with respect to the topology $\sigma(\mathcal{P}(E), B(E))$. An alternative expression for the divergence induced by $\rho$ is through the measure acceptance set

$$
\mathcal{A}:=\left\{P \circ X^{-1}: X \in L^{\infty}, \rho(X) \leq 0\right\} \subset \mathcal{P}(\mathbb{R}) .
$$

Indeed, we may then write

$$
\alpha(v \mid \mu)=\sup \left\{\int_{E} f d v: f \in B(E), \mu \circ f^{-1} \in \mathcal{A}\right\} .
$$

Divergences satisfy a consistency property related to (2.3), the statement of which requires some notation involving kernels. Given Polish spaces $E$ and $F$, a kernel from $E$ to $F$ is a measurable function $E \ni x \mapsto K_{x} \in$ $\mathcal{P}(F)$. Given $\mu \in \mathcal{P}(E)$, write $\mu K:=\int_{E} \mu(d x) K_{x}(\cdot)$ for the mean measure in $\mathcal{P}(F)$, i.e.,

$$
\mu K(A)=\int_{E} \mu(d x) K_{x}(A) \text {, for } A \subset F \text { Borel. }
$$

For $f \in B(F)$, write $K f$ for the function $K f(x)=\int_{F} K_{x}(d y) f(y)$ in $B(E)$. Note the identity $\int_{F} f d(\mu K)=\int_{E} K f d \mu$.

Proposition 2.6. Let $\rho$ be a law invariant risk measure with divergence $\alpha$. If $E$ and $F$ are Polish spaces and $K$ is a kernel from $E$ to $F$, then

$$
\alpha(\nu K \mid \mu K) \leq \alpha(v \mid \mu)
$$

for all $\mu, v \in \mathcal{P}(E)$. In particular, if $T: E \rightarrow F$ is measurable, then

$$
\alpha\left(v \circ T^{-1} \mid \mu \circ T^{-1}\right) \leq \alpha(v \mid \mu),
$$

and equality holds if $T$ is bijective with measurable inverse.

Proof. Note that the second claim follows from the first by setting $K(x, d y)=\delta_{T(x)}(d y)$. Jensen's inequality shows easily that $\mu \circ(K f)^{-1} \leq(\mu K) \circ f^{-1}$ in convex order for all $f \in B(F)$; indeed, for every convex function $\phi$ on $\mathbb{R}$,

$$
\int_{\mathbb{R}} \phi d \mu \circ(K f)^{-1}=\int_{E} \phi(K f) d \mu \leq \int_{E} K(\phi \circ f) d \mu=\int_{F} \phi \circ f d(\mu K)=\int_{\mathbb{E}} \phi d(\mu K) \circ f^{-1} .
$$

It is well known that (normalized) law invariant risk measures are increasing with respect to convex order, e.g. by [18, Corollary 4.65], and thus $\rho_{\mu K}(f) \geq \rho_{\mu}(K f)$. Then

$$
\begin{aligned}
\alpha(v K \mid \mu K) & =\sup _{f \in B(F)}\left(\int_{F} f d(v K)-\rho_{\mu K}(f)\right) \\
& =\sup _{f \in B(F)}\left(\int_{E}(K f) d \mu-\rho_{\mu K}(f)\right) \\
& \leq \sup _{g \in B(E)}\left(\int_{E} g d \mu-\rho_{\mu}(g)\right) \\
& =\alpha(v \mid \mu) .
\end{aligned}
$$


In fact, the inequality (2.5) is enough to reconstruct from $\alpha$ the original family of risk measures. This is made precise in the following:

Theorem 2.7. Suppose we are given family of functions $\mathcal{P}(E) \ni v \mapsto \alpha(v \mid \mu) \in[0, \infty]$, for each Polish space $E$ and each $\mu \in \mathcal{P}(E)$, and suppose the following conditions hold:

1. $\alpha(\mu \mid \mu)=0$.

2. $\alpha(v \mid \mu)=\infty$ if $\nu \in \mathcal{P}(E)$ is not absolutely continuous with respect to $\mu$.

3. $\alpha(v K \mid \mu K) \leq \alpha(v \mid \mu)$ for every $v \in \mathcal{P}(E)$ and every kernel $K$ from $E$ to another Polish space $F$.

For each Polish space E and each $\mu \in \mathcal{P}(E)$, define

$$
\rho_{\mu}(f):=\sup _{v \in \mathcal{P}(E)}\left(\int_{E} f d v-\alpha(v \mid \mu)\right), f \in B(E) .
$$

Then each $\rho_{\mu}$ is a law invariant risk measure. Moreover, for any Polish spaces $F$ and $G$, any $\mu \in \mathcal{P}(F)$ and $v \in \mathcal{P}(G)$, and any $f \in B(F)$ and $g \in B(G)$ with $\mu \circ f^{-1}=v \circ g^{-1}$, we have $\rho_{\mu}(f)=\rho_{\nu}(g)$.

Proof. It is immediate from the definition that $\rho_{\mu}$ is a risk measure. Indeed, since $\alpha(\mu \mid \mu)=0$ and $\alpha(v \mid \mu) \geq 0$ for all $v$, we have $\rho_{\mu}(0)=0$. Theorem 4.33 of [18] shows that $\rho_{\mu}$ satisfies the Fatou property, since the supremum in its definition includes only countably additive measures. For a fixed $\mu$, we deduce from property (3) and Proposition 2.4 that $\rho_{\mu}$ is law-invariant.

It remains to prove the last claim. Suppose for the moment that we can find a kernel $K$ from $F$ to $G$ such that $\mu K=v$ and $\mu(K g=f)=1$. Then

$$
\begin{aligned}
\rho_{\nu}(g) & =\sup _{\eta \in \mathcal{P}(G)}\left(\int_{G} g d \eta-\alpha(\eta \mid v)\right) \\
& \geq \sup _{\eta \in \mathcal{P}_{\mu}(F)}\left(\int_{G} g d(\eta K)-\alpha(\eta K \mid v)\right) \\
& =\sup _{\eta \in \mathcal{P}_{\mu}(F)}\left(\int_{F} f d \eta-\alpha(\eta K \mid \mu K)\right) \\
& \geq \sup _{\eta \in \mathcal{P}_{\mu}(F)}\left(\int_{F} f d \eta-\alpha(\eta \mid \mu)\right) \\
& =\rho_{\mu}(f) .
\end{aligned}
$$

Indeed, the second inequality follows from the assumption (3). Reversing the roles of $f$ and $g$ completes the proof. To prove the existence of such a kernel, we appeal to a famous theorem of Strassen [36, Theorem 3]: Define $h_{\phi}$ on $F$ by

$$
h_{\phi}(x)=\sup _{\eta \in S(x)} \int_{G} \phi d \eta, \quad \text { where } \quad S(x):=\left\{\eta \in \mathcal{P}(G): \int_{G} g d \eta=f(x)\right\} .
$$

If $S(x)$ is nonempty for each $x \in F$, and if $h_{\phi}$ is measurable, then Strassen's theorem says that there exists a kernel $K$ from $F$ to $G$ satisfying both $\mu K=v$ and $\mu(K g=f)=1$ if and only if

$$
\int_{G} \phi d v \leq \int_{F} h_{\phi} d \mu \text {, for all } \phi \in C_{b}(G) .
$$


Suppose for the moment that $S(x)$ is nonempty for each $x \in F$ and that $h_{\phi}$ is measurable, so that we can apply this theorem. Define a new function $\widetilde{h}_{\phi}$ on $\mathbb{R}$ by

$$
\widetilde{h}_{\phi}(a):=\sup \phi\left(g^{-1}(\{a\})\right)=\sup \{\phi(y): y \in G, g(y)=a\},
$$

with the usual convention $\sup \emptyset=-\infty$. Let us check that

$$
\widetilde{h}_{\phi}(f(x)) \leq h_{\phi}(x), \mu \text {-a.e. } x \in F .
$$

If $x \in F$ has $\widetilde{h}_{\phi}(f(x))=-\infty$, there is nothing to prove. So suppose $x \in F$ satisfies $\widetilde{h}_{\phi}(f(x))>-\infty$. For fixed $\epsilon>0$ we may then choose $y_{0} \in G$ such that $g\left(y_{0}\right)=f(x)$ and $\phi\left(y_{0}\right) \geq \widetilde{h}_{\phi}(f(x))-\epsilon$. Then, since $\int_{G} g d \delta_{y_{0}}=f(x)$, we have by definition $\phi\left(y_{0}\right) \leq h_{\phi}(x)$, and thus $\widetilde{h}_{\phi}(f(x)) \leq \epsilon+h_{\phi}(x)$. Since $\epsilon$ was arbitrary, this proves (2.8). Finally, since clearly $\phi(x) \leq \widetilde{h}_{\phi}(g(x))$ for all $x \in G$, using $v \circ g^{-1}=\mu \circ f^{-1}$ we prove (2.7):

$$
\int_{G} \phi d v \leq \int_{G} \widetilde{h}_{\phi} \circ g d v=\int_{F} \widetilde{h}_{\phi} \circ f d \mu \leq \int_{F} h_{\phi} d \mu .
$$

It remains to check the technical points left out above. First note that $S(x)$ is nonempty for $\mu$-almost every $x$ : If $S(x)$ is empty then $f(x)$ is not in the range of $g$, which cannot hold on a set of positive $\mu$-measure because $\mu \circ f^{-1}=v \circ g^{-1}$. Modify $S(x)$ to equal $F$ on a null set in such a way that $S(x) \neq \emptyset$ for all $x$. Next note that $h_{\phi}$ is universally measurable because the graph of $S(x)$ is analytic [9, Proposition 7.47], so we may apply Strassen's theorem by simply replacing the Borel $\sigma$-field on $F$ with its universal completion.

With the previous result in mind, it is natural to make the following definition:

Definition 2.8. A divergence is a family of convex lower-semicontinuous (with respect to total variation) functions $\mathcal{P}(E) \ni v \mapsto \alpha(v \mid \mu) \in[0, \infty]$, for each Polish space $E$ and each $\mu \in \mathcal{P}(E)$, satisfying properties (1-3) of Theorem 2.7. Given a divergence $\alpha$, the corresponding (or induced) family of risk measures is the family $\left(\rho_{\mu}\right)_{\mu, E}$ defined by (2.6). The corresponding (or induced) risk measure is the risk measure $\bar{\rho}$ defined on $L^{\infty}=$ $L^{\infty}(\Omega, \mathcal{F}, P)$ by

$$
\bar{\rho}(X):=\rho_{P \circ X^{-1}}(i d),
$$

where id denotes the identity map on $\mathbb{R}$. Thanks to Theorem $2.7, \bar{\rho}$ is well defined. It is straightforward to check that its induced divergence is exactly $\alpha$, and also that $\bar{\rho}_{\mu}=\rho_{\mu}$ for each Polish space $E$ and $\mu \in \mathcal{P}(E)$.

Before we proceed, we state here a technical but important observation that we will use later:

Lemma 2.9. Let $\rho$ be a law invariant risk measure. Fix a Polish space $E$ and a function $f \in B(E)$. The map $\Phi: \mathcal{P}(E) \rightarrow \mathbb{R}$ given by $\Phi(\mu):=\rho_{\mu}(f)$ is measurable. Moreover, if $f \in C_{b}(E)$, then $\Phi$ is lower semicontinuous.

Proof. First we prove the second claim. Let $\mu_{n} \rightarrow \mu$ in $\mathcal{P}(E)$. By Skorohod representation, we may find $E$ valued random variables $X, X_{n}$ defined on $\Omega$ with $P \circ X^{-1}=\mu, P \circ X_{n}^{-1}=\mu_{n}$, and $X_{n} \rightarrow X$ almost surely. Then $f\left(X_{n}\right) \rightarrow f(X)$ almost surely since $f$ is continuous, and the sequence $f\left(X_{n}\right)$ is uniformly bounded. Thus, the Fatou property (Theorem 2.1) implies

$$
\rho_{\mu}(f)=\rho(f(X)) \leq \liminf _{n \rightarrow \infty} \rho\left(f\left(X_{n}\right)\right)=\liminf _{n \rightarrow \infty} \rho_{\mu_{n}}(f) .
$$

To prove the first claim, find $M>0$ such that $|f| \leq M$, and write $\rho_{\mu}(f)=\rho_{\mu \circ f^{-1}}(i d)$, where $i d$ denotes the identity map on $[-M, M]$. According to the previous argument, $m \mapsto \rho_{m}(i d)$ is lower semicontinuous and thus measurable on $\mathcal{P}([-M, M])$. Since also $\mu \mapsto \mu \circ f^{-1}$ is Borel measurable from $\mathcal{P}(E)$ to $\mathcal{P}([-M, M])$ (easily proven using, e.g., [9, Proposition 7.25]), we see that $\Phi$ is the composition of two measurable maps. 


\subsection{Simplified divergences}

An important property of relative entropy is that its dual formula can be reduced to a supremum over continuous functions: For a Polish space $E$ and for $\mu, v \in \mathcal{P}(E)$,

$$
H(v \mid \mu)=\sup _{f \in B(E)}\left(\int_{E} f d v-\log \int_{E} e^{f} d \mu\right)=\sup _{f \in C_{b}(E)}\left(\int_{E} f d v-\log \int_{E} e^{f} d \mu\right) .
$$

Such a simplification is not always possible and motivates the identification of a nice class of divergences:

Definition 2.10. A divergence $\alpha$ is said to be simplified if, for every $\mu \in \mathcal{P}([0,1])$, the function $\alpha(\cdot \mid \mu)$ is weakly lower semicontinuous on $\mathcal{P}([0,1])$, where "weakly" refers to the usual weak convergence topology $\sigma(\mathcal{P}([0,1]), C([0,1]))$.

Our characterization of superadditivity of divergences in Section 5 is notably restricted to the class of simplified divergences, essentially because this property ensures that the functional of $v(d x) K_{x}^{v}(d y) \in \mathcal{P}(E \times F)$ appearing in the right-hand side of (1.2) is lower semicontinuous. This additional assumption is admittedly a nuisance, but it does not seem that we can do without it in Section 5, and the related work of [5] imposes a similar condition. Before we proceed, we state some equivalent forms:

Proposition 2.11. Let $\alpha$ be a divergence, and let $\rho$ be the corresponding risk measure. The following are equivalent:

(1) $\alpha$ is simplified.

(2) For each $\mu, v \in \mathcal{P}([0,1])$ we have

$$
\alpha(v \mid \mu)=\sup _{f \in C([0,1])}\left(\int_{[0,1]} f d v-\rho_{\mu}(f)\right) .
$$

(3) For $f_{n} \in C([0,1])$ and $f \in B([0,1])$ with $f_{n} \downarrow f$ pointwise, we have $\rho_{\mu}\left(f_{n}\right) \downarrow \rho_{\mu}(f)$.

Proof. Equivalence of (1) and (2) is a simple application of the Fenchel-Moreau theorem: Let $M([0,1])$ denote the set of bounded finitely additive signed measures on $[0,1]$, and extend $\alpha(\cdot \mid \mu)$ to $M([0,1])$ by setting $\bar{\alpha}_{\mu}(v)=$ $\alpha(v \mid \mu)$ for $v \in \mathcal{P}([0,1])$ and $\bar{\alpha}_{\mu}(v)=\infty$ otherwise. Put $M([0,1])$ and $C([0,1])$ in duality. Then $\rho_{\mu}$ is the convex conjugate of $\bar{\alpha}_{\mu}$. As $\bar{\alpha}_{\mu}$ is convex, proper, and lower semicontinuous, it must equal its biconjugate.

To see why (1) and (3) are equivalent, we note first that (1) is equivalent to weak compactness of the sublevel sets of $\alpha(\cdot \mid \mu)$, simply because $\mathcal{P}([0,1])$ is weakly compact. The equivalence of (1) and (3) now follows from [5, Theorem 2.4], noting that $\rho_{\mu}(\cdot)$ automatically has the Fatou property by Theorem 2.1 .

One other useful feature of simplified divergences is the following measurability result, which we are unfortunately unable to prove without the additional assumption.

Lemma 2.12. Every simplified divergence $\alpha$ is jointly measurable, in the sense that for any fixed Polish space $E$ the function $\alpha(\cdot \mid \cdot)$ is jointly measurable on $\mathcal{P}(E) \times \mathcal{P}(E)$ (with respect to the Borel $\sigma$-field generated by the topology of weak convergence).

Proof. Fix a Polish space $E$. By Borel isomorphism (see [25, Theorem 15.6]), there exists a measurable bijection $T: E \rightarrow[0,1]$ with measurable inverse. It follows from Proposition 2.6 that

$$
\alpha\left(\nu \circ T^{-1} \mid \mu \circ T^{-1}\right)=\alpha(v \mid \mu),
$$

for all $\mu, v \in \mathcal{P}(E)$. Note also that the map $\mu \mapsto \mu \circ T^{-1}$ is a measurable bijection from $\mathcal{P}(E)$ to $\mathcal{P}([0,1])$ with measurable inverse. Thus, to show $\alpha$ is jointly measurable on $\mathcal{P}(E) \times \mathcal{P}(E)$, it suffices to show it is jointly 
measurable on $\mathcal{P}([0,1]) \times \mathcal{P}([0,1])$. Since $\alpha$ is simplified, we have

$$
\alpha(v \mid \mu)=\sup _{f \in C([0,1])}\left(\int f d v-\rho_{\mu}(f)\right)
$$

for $\mu, v \in \mathcal{P}([0,1])$. Since $\rho_{\mu}$ is Lipschitz with respect to the supremum norm on $C([0,1])$, and since $C([0,1])$ is separable, we may reduce the supremum above to a countable one. But $v \mapsto \int f d v$ is measurable for each $f \in C([0,1])$, as is $\mu \mapsto \rho_{\mu}(f)$ thanks to Lemma 2.9.

\section{Joint lower semicontinuity of divergences}

This section studies lower semicontinuity properties of $\alpha$, in part for their intrinsic interest, and in part for another tractable condition that will allow us to verify that all of the examples of divergences we discuss in Section 7 are indeed simplified in the sense of Definition 2.10. We know that for any divergence $\alpha$, the map $\alpha(\cdot \mid \mu)$ is lower semicontinuous with respect to the topology $\sigma(\mathcal{P}(E), B(E))$ for any fixed $\mu, E$. We begin by showing that this can always be strengthened to joint lower semicontinuity in the same topology. Note that Lemma 2.12 does not follow from the following Proposition 3.1, because the Borel $\sigma$-field of $\sigma(\mathcal{P}(E), B(E))$ is typically strictly larger than the Borel $\sigma$-field of the topology of weak convergence.

Proposition 3.1. Suppose $\rho$ is a law invariant risk measure with induced divergence $\alpha$. If $\mathcal{P}(E)$ is endowed with the topology $\sigma\left(\mathcal{P}(E), B(E)\right.$ ), then the map $\mu \mapsto \rho_{\mu}(f)$ is continuous for every $f \in B(E)$, and $\alpha(\cdot \mid \cdot)$ is lower semicontinuous with respect to the product topology on $\mathcal{P}(E) \times \mathcal{P}(E)$.

Proof. First, fix $f \in B(E)$ with $|f| \leq M$ for $M>0$. Note that $\rho_{\mu}(f)=\rho_{\mu \circ f^{-1}}(i d)$, where id denotes the identity map on $[-M, M]$. We saw in Lemma 2.9 that $\mathcal{P}([-M, M]) \ni m \mapsto \rho_{m}(f)$ is continuous with respect to weak convergence. It is easy to check that $\mu \mapsto \mu \circ f^{-1}$ is a continuous map from $(\mathcal{P}(E), \sigma(\mathcal{P}(E), B(E)))$ to $\mathcal{P}([-M, M])$ endowed with the weak convergence topology, and this proves the first claim. According to the definition (2.4), $\alpha(v \mid \mu)$ is the supremum of continuous functions of $(v, \mu)$, and this proves the second claim.

On the other hand, relative entropy is known to be jointly lower semicontinuous with respect to weak convergence, i.e., the topology $\sigma\left(\mathcal{P}(E), C_{b}(E)\right)$. We next characterize those divergences which share this property. For this it helps to make two definitions, the second of which is well known:

Definition 3.2. We say a divergence $\alpha$ is jointly weakly lower semicontinuous if, for each Polish space $E$, the map $\alpha(\cdot \mid \cdot)$ is lower semicontinuous on $\mathcal{P}(E) \times \mathcal{P}(E)$ with respect to the topology of weak convergence, i.e., equipping $\mathcal{P}(E)$ with the topology $\sigma\left(\mathcal{P}(E), C_{b}(E)\right)$.

Definition 3.3. We say a risk measure $\rho$ is Lebesgue continuous if whenever $X_{n} \in L^{\infty}$ is a uniformly bound sequence with $X_{n} \rightarrow X$ a.s. we have $\rho\left(X_{n}\right) \rightarrow \rho(X)$. This is equivalent to the seemingly weaker condition that whenever $X_{n}, X \in L^{\infty}$ with $X_{n} \downarrow X$ a.s. we have $\rho\left(X_{n}\right) \downarrow \rho(X)$ (c.f. Remark 4.25 and Exercise 4.2.2 of [18]).

The main result of this section is the following characterization of joint lowercontinuity:

Theorem 3.4. Let $\rho$ be a law invariant risk measure with induced divergence $\alpha$. The following are equivalent:

(1) $\alpha$ is jointly weakly lower semicontinuous.

(2) For each Polish space $E$ and each $f \in C_{b}(E)$, the map $\mu \mapsto \rho_{\mu}(f)$ is continuous.

(3) For each Polish space E, each $\mu \in \mathcal{P}(E)$, and each $f, f_{n} \in C_{b}(E)$ with $f_{n} \rightarrow$ f pointwise and with $f_{n}$ uniformly bounded, we have $\rho_{\mu}\left(f_{n}\right) \rightarrow \rho_{\mu}(f)$.

(4) $\rho$ is Lebesgue continuous. 
If these conditions hold, then:

(5) For each Polish space E and each $v, \mu \in \mathcal{P}(E)$ we have

$$
\alpha(v \mid \mu)=\sup _{f \in C_{b}(E)}\left(\int_{E} f d v-\rho_{\mu}(f)\right) .
$$

Proof. $(1 \Rightarrow 2)$ Suppose first that $E$ is compact. Let $\mu_{n} \rightarrow \mu$ in $\mathcal{P}(E)$. We know from Lemma 2.9 that $\rho_{\mu}(f) \leq$ $\liminf _{n \rightarrow \infty} \rho_{\mu_{n}}(f)$, so we show upper semicontinuity. Let $\epsilon>0$, and find for each $n$ some $v_{n} \in \mathcal{P}(E)$ satisfying

$$
\rho_{\mu_{n}}(f) \leq \epsilon+\int f d v_{n}-\alpha\left(v_{n} \mid \mu_{n}\right) .
$$

Since $E$ is compact, every subsequence admits a further subsequence $\left\{n_{k}\right\}$ such that $v_{n_{k}} \rightarrow v$ for some $v \in$ $\mathcal{P}(E)$, and lower semicontinuity of $\alpha$ implies

$$
\limsup _{k \rightarrow \infty} \rho_{\mu_{n_{k}}}(f) \leq \epsilon+\int f d v-\alpha(v \mid \mu) \leq \epsilon+\rho_{\mu}(f) .
$$

This shows $\lim \sup _{n \rightarrow \infty} \rho_{\mu_{n}}(f) \leq \rho_{\mu}(f)$. Finally, if $E$ is not necessarily compact, find $M>0$ such that $\mu_{n}(|f| \leq$ $M)=1$. Since $[-M, M]$ is compact, the previous result shows that

$$
\rho_{\mu_{n}}(f)=\rho_{\mu_{n} \circ f^{-1}}(i d) \rightarrow \rho_{\mu \circ f^{-1}}(i d)=\rho_{\mu}(f),
$$

where id is the identity map on $[-M, M]$.

(2 $\Rightarrow 3$ ) Let $f_{n}, f \in C_{b}(E)$ be uniformly bounded with $f_{n} \rightarrow f \mu$-a.s. Then there exists $M>0$ such that $\mu\left(\left|f_{n}\right| \leq M\right)=1$ for all $n$, and so

$$
\rho_{\mu}\left(f_{n}\right)=\rho_{\mu \circ f_{n}^{-1}}(i d) \rightarrow \rho_{\mu \circ f^{-1}}(i d)=\rho_{\mu}(f),
$$

where id denotes the identity map on $[-M, M]$.

( $3 \Rightarrow 4$ ) Let $X, X_{n} \in L^{\infty}$ be uniformly bounded with $X_{n} \rightarrow X$ a.s. Find $M>0$ such that $\left|X_{n}\right| \leq M$ a.s. for all $n$. Let $E$ denote the (complete separable) metric space of convergent sequences with values in [ $-M, M]$ endowed with the supremum metric, and define $\mu$ on $E$ by

$$
\mu=P \circ\left(X_{1}, X_{2}, \ldots\right)^{-1} .
$$

Let $f_{n}(x)=x_{n}$ denote the coordinate maps, and let $f(x)=\lim _{n \rightarrow \infty} x_{n}$, for $x=\left(x_{1}, x_{2}, \ldots\right) \in E$. Then $f$ and $f_{n}$ are uniformly bounded and continuous, with $f_{n} \rightarrow f$ pointwise by construction. Since $\mu \circ f_{n}^{-1}=P \circ X_{n}^{-1}$ and $\mu \circ f^{-1}=P \circ X^{-1}$, we have

$$
\rho\left(X_{n}\right)=\rho_{\mu}\left(f_{n}\right) \rightarrow \rho_{\mu}(f)=\rho(X) .
$$

$(4 \Rightarrow 5)$ Clearly we have

$$
\alpha(v \mid \mu) \geq \sup _{f \in C_{b}(E)}\left(\int f d v-\rho_{\mu}(f)\right) .
$$

To show the reverse inequality, fix $\epsilon>0$ and find $f \in B(E)$ such that

$$
\alpha(v \mid \mu) \leq \epsilon+\int f d v-\rho_{\mu}(f)
$$

Find a bounded sequence $f_{n}$ of continuous functions with $f_{n} \rightarrow f$ a.s. Then, using (3) and the bounded convergence theorem, we get

$$
\alpha(v \mid \mu) \leq \epsilon+\lim _{n \rightarrow \infty}\left(\int f_{n} d v-\rho_{\mu}\left(f_{n}\right)\right) \leq \epsilon+\sup _{f \in C_{b}(E)}\left(\int f d v-\rho_{\mu}(f)\right) .
$$


$(4 \Rightarrow 3)$ Obvious.

$(3 \Rightarrow 2)$ Let $\mu_{n} \rightarrow \mu$ in $\mathcal{P}(E)$ and $f \in C_{b}(E)$. Let $\lambda$ denote Lebesgue measure on [0,1], and let $q_{n}$ and $q$ denote the quantile functions corresponding to $\mu_{n} \circ f^{-1}$ and $\mu \circ f^{-1}$, respectively, so that $\mu_{n} \circ f^{-1}=\lambda \circ q_{n}^{-1}$ and $\mu \circ f^{-1}=\lambda \circ q^{-1}$. Then $q_{n}$ are uniformly essentially bounded with $q_{n} \rightarrow q \lambda$-a.s., and law invariance yields

$$
\rho_{\mu_{n}}(f)=\rho_{\lambda}\left(q_{n}\right) \rightarrow \rho_{\lambda}(q)=\rho_{\mu}(f) .
$$

( $4 \Rightarrow 1$ ) We know by now that (4) implies both (5) and (2), and thus we can write

$$
\alpha(v \mid \mu)=\sup _{f \in C_{b}(E)}\left(\int f d v-\rho_{\mu}(f)\right) .
$$

Since the map $(\mu, v) \mapsto \int f d v-\rho_{\mu}(f)$ is jointly continuous by (2), $\alpha$ is lower semicontinuous as the supremum of continuous functions.

\section{Joint convexity of divergences}

While every divergence is convex in its first argument by definition, it is well known that relative entropy and also $f$-divergences are jointly convex. It turns out that joint convexity of a divergence is equivalent to concavity of the corresponding law invariant risk measure on the level of distributions. To be clear, for a law invariant risk measure $\rho$, define the function $\tilde{\rho}$ on the set of probability measures on $\mathbb{R}$ with compact support by setting $\tilde{\rho}\left(P \circ X^{-1}\right)=\rho(X)$, for $X \in L^{\infty}$. The concavity of $\tilde{\rho}$ was studied recently by Acciaio and Svindland [2], who make a compelling case that concavity is much more common in spite of the convexity of $\rho$ on the level of random variables. Indeed, they show that $\rho(X)=\mathbb{E} X$ is the only law invariant risk measure for which $\tilde{\rho}$ is convex. The entropic risk measure, for example, clearly has $\tilde{\rho}$ concave. Moreover, if $\rho$ is the optimized certainty equivalent corresponding to a function $\phi$, then the formula

$$
\tilde{\rho}(\mu)=\inf _{m \in \mathbb{R}}\left(\int \phi(m+x) \mu(d x)-m\right)
$$

shows that $\tilde{\rho}$ is concave.

Proposition 4.1. Let $\rho$ be a law invariant risk measure with induced divergence $\alpha$. The following are equivalent:

(1) $\alpha$ is jointly convex, in the sense that $\alpha(\cdot \cdot \cdot)$ is convex on $\mathcal{P}(E) \times \mathcal{P}(E)$ for each Polish space $E$.

(2) For each Polish space $E$ and each $f \in B(E)$, the map $\mu \mapsto \rho_{\mu}(f)$ is concave.

(3) $\tilde{\rho}$ is concave.

Proof. $(1 \Rightarrow 2)$ Let $E$ be a Polish space and $f \in B(E)$. Fix $t \in(0,1)$ and $\mu_{1}, \mu_{2} \in \mathcal{P}(E)$. Then (1) implies

$$
\begin{aligned}
\rho_{t \mu_{1}+(1-t) \mu_{2}}(f) & =\sup _{v \in \mathcal{P}(E)}\left\{\int f d v-\alpha\left(v \mid t \mu_{1}+(1-t) \mu_{2}\right)\right\} \\
& \geq \sup _{v_{1}, v_{2} \in \mathcal{P}(E)}\left\{t \int f d v_{1}+(1-t) \int f d v_{2}-\alpha\left(t v_{1}+(1-t) v_{2} \mid t \mu_{1}+(1-t) \mu_{2}\right)\right\} \\
& \geq \sup _{v_{1}, v_{2} \in \mathcal{P}(E)}\left\{t \int f d v_{1}+(1-t) \int f d v_{2}-t \alpha\left(v_{1} \mid \mu_{1}\right)+(1-t) \alpha\left(v_{2} \mid \mu_{2}\right)\right\} \\
& =t \rho_{\mu_{1}}(f)+(1-t) \rho_{\mu_{2}}(f) .
\end{aligned}
$$


( $2 \Rightarrow 1$ ) On the other hand, if $v_{1}, v_{2} \in \mathcal{P}(E)$, then (2) implies

$$
\begin{aligned}
\alpha\left(t v_{1}+(1-t) v_{2} \mid t \mu_{1}+(1-t) \mu_{2}\right) & =\sup _{f \in B(E)}\left\{t \int f d v_{1}+(1-t) \int f d v_{2}-\rho_{t \mu_{1}+(1-t) \mu_{2}}(f)\right\} \\
& \leq \sup _{f \in B(E)}\left\{t \int f d v_{1}+(1-t) \int f d v_{2}-t \rho_{\mu_{1}}(f)+(1-t) \rho_{\mu_{2}}(f)\right\} \\
& \leq t \alpha\left(v_{1} \mid \mu_{1}\right)+(1-t) \alpha\left(v_{2} \mid \mu_{2}\right) .
\end{aligned}
$$

$(3 \Rightarrow 2)$ This is immediate from the identity

$$
\rho_{t \mu_{1}+(1-t) \mu_{2}}(f)=\tilde{\rho}\left(t \mu_{1} \circ f^{-1}+(1-t) \mu_{2} \circ f^{-1}\right) .
$$

$\left(2 \Rightarrow 3\right.$ ) This is almost immediate from the above identity. Assume (2). Let $m_{1}, m_{2} \in \mathcal{P}(\mathbb{R})$ have compact support, and let $t \in(0,1)$. Then, letting id denote the identity map on $\mathbb{R}$,

$$
\tilde{\rho}\left(t m_{1}+(1-t) m_{2}\right)=\rho_{t m_{1}+(1-t) m_{2}}(i d) \geq t \rho_{m_{1}}(i d)+(1-t) \rho_{m_{2}}(i d)=t \tilde{\rho}\left(m_{1}\right)+(1-t) \tilde{\rho}\left(m_{2}\right) .
$$

\section{Acceptance consistency and superadditivity}

As was first observed by Weber [38], a law invariant risk measure naturally gives rise to a dynamic riskmeasure on any (nice enough) filtered probability space. We will use the same construction: Define $\tilde{\rho}$ again by $\tilde{\rho}(P$ 。 $\left.X^{-1}\right)=\rho(X)$, which makes sense thanks to law-invariance. Using our previous notation, note that $\tilde{\rho}(m)=$ $\rho_{m}(i d)$, where id denotes the identity map on $\mathbb{R}$. We may then define, for any $\sigma$-field $\mathcal{G} \subset \mathcal{F}$ in $\Omega$ and any $X \in L^{\infty}$,

$$
\rho(X \mid \mathcal{G}):=\tilde{\rho}(P(X \in \cdot \mid \mathcal{G})) .
$$

Note that a regular conditional law of $X$ given $\mathcal{G}$ exists because $\Omega$ is standard. Lemma 2.9 ensures that $\rho(X \mid \mathcal{G})$ is a $\mathcal{G}$-measurable random variable, defined uniquely up to a.s. equality. Similarly, for a random variable $Y$, write $\rho(X \mid Y):=\rho(X \mid \sigma(Y))$. Note that if $Y$ is $\mathcal{G}$-measurable then

$$
\rho(X+Y \mid \mathcal{G})=\rho(X \mid \mathcal{G})+Y, \text { a.s. }
$$

for any random variable $X$. If $X$ and $Y$ are independent, then it is straightforward to check that

$$
\rho(f(X, Y) \mid X)=\left.\rho(f(x, Y))\right|_{X=X} .
$$

We are nearly ready to define the type of time-consistency we investigate.

Definition 5.1. We say that a law-invariant risk measure $\rho$ is acceptance consistent if

$$
\rho(X) \leq \rho(\rho(X \mid \mathcal{G})),
$$

for all sub- $\sigma$-fields $\mathcal{G} \subset \mathcal{F}$ and all $X \in L^{\infty}$. If the inequality is reversed, we say $\rho$ is rejection consistent. We say $\rho$ is time consistent if it is both acceptance and rejection consistent.

Remark 5.2. This definition begins to look more like the one appearing in the literature (see [1]) once it is applied inductively. Let $\left(\mathcal{F}_{t}\right)_{t \geq 0}$ denote any filtration on $\Omega$, with $\mathcal{F}_{t} \subset \mathcal{F}$ for all $t$. Indeed, $\left(\rho\left(\cdot \mid \mathcal{F}_{t}\right)\right)_{t \geq 0}$ is a dynamic risk measure in the sense of [1]. If $\rho$ is acceptance consistent and $X \in L^{\infty}$, then it is straightforward to check that $\rho\left(X \mid \mathcal{F}_{s}\right) \leq \rho\left(\rho\left(X \mid \mathcal{F}_{t}\right) \mid \mathcal{F}_{s}\right)$ a.s. for $0 \leq s \leq t$. 


\subsection{Superadditivity and shift-convexity}

Let us give names to certain divergence inequalities resembling the chain rule of classical relative entropy. Henceforth we will need to assume that our divergences are simplified, as in Definition 2.10. As far as the following definition of superadditivity is concerned, this assumption is merely to ensure that the divergence $\alpha(\cdot \mid \cdot)$ is jointly measurable, so that the integrals make sense. Later, a technical point in the proof of the main Theorem 5.5 will depend crucially on the divergence being simplified, but the question of whether or not Theorem 5.5 holds in more generality remains open.

Definition 5.3. We say that a divergence $\alpha$ is partially superadditive (resp. partially subadditive) if

$$
\alpha\left(v(d x) K_{x}^{v}(d y) \mid \mu_{1} \times \mu_{2}\right) \geq \alpha\left(v \mid \mu_{1}\right)+\int v(d x) \alpha\left(K_{x}^{v} \mid \mu_{2}\right), \quad(\text { resp. } \leq)
$$

whenever $v(d x) K_{x}^{v}(d y)$ and $\mu_{1} \times \mu_{2}$ are probability measures on the product of two Polish spaces; note that the latter is required to be a product measure. We say a simplified divergence $\alpha$ is (fully) superadditive (resp. subadditive) if

$$
\alpha\left(v(d x) K_{x}^{v}(d y) \mid \mu(d x) K_{x}^{\mu}(d y)\right) \geq \alpha(v \mid \mu)+\int v(d x) \alpha\left(K_{x}^{v} \mid K_{x}^{\mu}\right), \quad(\text { resp. } \leq)
$$

whenever $v(d x) K_{x}^{v}(d y)$ and $\mu(d x) K_{x}^{\mu}(d y)$ are probability measures on the product of two Polish spaces.

Note that partial superadditivity as opposed to full superadditivity only requires the inequality to hold when the reference measure is a product. It turns out that these conditions are equivalent, although we have only an indirect proof of this fact. As was discussed in the introduction, additivity properties of a divergence $\alpha$ are linked with time consistency and sub-level set properties of its induced risk measure, which we now describe. In the following, we will write $\mathcal{P}[-M, M]$ for $M>0$ for the set of probability measures on $\mathbb{R}$ which are supported on the interval $[-M, M]$.

\section{Definition 5.4.}

1. The measure acceptance set $\mathcal{A}$ of a law invariant risk measure $\rho$ is defined by $\mathcal{A}:=\left\{P \circ X^{-1}: X \in\right.$ $\left.L^{\infty}, \rho(X) \leq 0\right\}$. In words, this is the set of laws of random variables $X$ satisfying $\rho(X) \leq 0$.

2. A set $\mathcal{A} \subset \mathcal{P}(\mathbb{R})$ is shift-convex if for every $\mu \in \mathcal{A}$, every $M>0$, and every measurable map $\mathbb{R} \ni x \mapsto K_{X} \in$ $\mathcal{A} \cap \mathcal{P}[-M, M]$, it holds that the measure $\int_{\mathbb{R}} \mu(d x) K_{x}(\cdot-x)$ is in $\mathcal{A}$.

As was discussed by Weber [38], the convexity of a measure acceptance set $\mathcal{A}$ admits a natural interpretation in terms of so-called compound lotteries: If two outcomes $X$ and $Y$ are acceptable, then convexity of $\mathcal{A}$ means that the outcome with law $U X+(1-U) Y$ is also acceptable, where $U$ is an independent Bernoulli $(\{0,1\}$-valued) random variable. Shift-convexity is open to interpretation on similar grounds: Suppose $X$ is an acceptable outcome, and that $Y$ is conditionally acceptable given $X$. Then shift-convexity means that $X+Y$ is itself acceptable. To see this, in the definition of shift-convexity take $\mu$ to be the law of $X$ and $K_{X}$ to be the conditional law of $Y$ given $X=x$. We can now state the main result of this section.

Theorem 5.5. Suppose $\alpha$ is a simplified divergence induced by a law invariant risk measure $\rho$ with acceptance set $\mathcal{A}$. The following are equivalent:

(1) $\rho$ is acceptance consistent.

(2) $\mathcal{A}$ is shift-convex.

(3) $\alpha$ is superadditive.

(4) $\alpha$ is partially superadditive. 
Similarly, the same equivalences hold when "acceptance" is changed to "rejection", "superadditive" is changed to "subadditive", and $\mathcal{A}$ is changed to $\mathcal{A}^{c}$. The equivalence of (1) and (2) holds without the assumption that $\alpha$ is simplified.

From Theorem 5.5 we can conclude that not many divergences are additive, i.e., both superadditive and subadditive. According to Kupper and Schachermayer [27], the only time consistent law invariant risk measures are entropic:

Corollary 5.6. Suppose $\alpha$ is a simplified divergence. If $\alpha$ is both superadditive and subadditive, then it is of one of the following forms:

$$
\begin{aligned}
& \alpha(v \mid \mu)=\frac{1}{\eta} H(v \mid \mu), \text { for some } \eta>0, \text { for } v \ll \mu, \quad \infty \text { otherwise, } \\
& \alpha(v \mid \mu)=0 \text { for } v=\mu, \quad \infty \text { otherwise, } \\
& \alpha(v \mid \mu)=0 \text { for } v \ll \mu, \quad \infty \text { otherwise. }
\end{aligned}
$$

The induced risk measures are $\rho(X)=\eta^{-1} \log \mathbb{E}\left[e^{\eta X}\right], \rho(X)=\mathbb{E} X$, and $\rho(X)=\operatorname{ess} \sup X$, respectively.

\subsection{Properties of time consistency}

The following lemma shows that acceptance consistency is equivalent to a seemingly weaker statement, which will be easier to connect with shift-convexity:

Lemma 5.7. Let $\rho$ be a law invariant risk measure. Then $\rho$ is acceptance consistent if and only if the following holds: For every pair of independent random variables $X, Y$ with values in some Polish spaces $E, F$, and for every $f \in B(E \times F)$, we have

$$
\rho(f(X, Y)) \leq \rho(\rho(f(X, Y) \mid X)) .
$$

Proof. The "only if" direction is immediate. To prove the converse, fix $X \in L^{\infty}$ and a $\sigma$-field $\mathcal{G} \subset \mathcal{F}$. Find $Y \in L^{\infty}$ which generates $\mathcal{G}$, for example $Y=\sum_{n=1}^{\infty} 2^{-n} 1_{B_{n}}$ where $\left\{B_{n}\right\}$ is a countable family of generators of $\mathcal{G}$ (recall that our ambient probability space is standard). By [24, Theorem 5.10], we may find independent random variables $\widetilde{Y}$ and $U$ as well as a measurable function $f$ such that $(\widetilde{Y}, f(\widetilde{Y}, U))$ has the same law as $(Y, Z)$. Then the hypothesis and law invariance imply

$$
\rho(Z)=\rho(f(\widetilde{Y}, U)) \leq \rho(\rho(f(\widetilde{Y}, U) \mid \widetilde{Y})) .
$$

But the conditional law of $f(\widetilde{Y}, U)$ given $\widetilde{Y}$ is the same as the conditional law of $Z$ given $Y$, and thus law invariance of $\rho$ implies that $\rho(f(\widetilde{Y}, U) \mid \widetilde{Y})$ and $\rho(Z \mid Y)=\rho(Z \mid \mathcal{G})$ have the same law. Using law invariance once more, we conclude that

$$
\rho(\rho(f(\widetilde{Y}, U) \mid \widetilde{Y}))=\rho(\rho(Z \mid \mathcal{G})) .
$$

The next lemma rephrases acceptance consistency in a more measure-theoretic notation which will be useful later.

Proposition 5.8. For a law invariant risk measure $\rho$, the following are equivalent:

(1) $\rho$ is acceptance consistent.

(2) For Polish spaces $E$ and $F, \bar{\mu}=\mu(d x) K_{x}^{\mu}(d y) \in \mathcal{P}(E \times F), f \in B(E \times F)$, and $g \in B(E)$ satisfying $\rho_{\mu}(g) \leq 0$, we have

$$
\mu\left\{x \in E: \rho_{K_{x}^{\mu}}(f(x, \cdot)) \leq g(x)\right\}=1 \quad \Rightarrow \quad \rho_{\bar{\mu}}(f) \leq 0 .
$$


(3) For Polish spaces $E$ and $F, \bar{\mu}=\mu(d x) K_{x}^{\mu}(d y) \in \mathcal{P}(E \times F)$, and $f \in B(E \times F)$, we have

$$
\rho_{\mu}\left(\left.\rho_{K_{x}^{\mu}}(f(x, \cdot))\right|_{X=X}\right) \geq \rho_{\bar{\mu}}(f),
$$

(4) For Polish spaces $E$ and $F, \mu_{1} \in \mathcal{P}(E), \mu_{2} \in \mathcal{P}(F), f \in B(E \times F)$, and $g \in B(E)$ satisfying $\rho_{\mu_{1}}(g) \leq 0$, we have

$$
\mu\left\{x \in E: \rho_{\mu_{2}}(f(x, \cdot)) \leq g(x)\right\}=1 \quad \Rightarrow \quad \rho_{\mu_{1} \times \mu_{2}}(f) \leq 0 .
$$

(5) For Polish spaces $E$ and $F, \bar{\mu}=\mu_{1} \times \mu_{2} \in \mathcal{P}(E \times F)$, and $f \in B(E \times F)$, we have

$$
\rho_{\mu_{1}}\left(\left.\rho_{\mu_{2}}(f(x, \cdot))\right|_{X=X}\right) \geq \rho_{\bar{\mu}}(f),
$$

where $X$ denotes the identity map on $E$.

The same equivalences hold for rejection consistent, but with the inequalities reversed.

Proof. It is obvious that (3) implies (5) and (2) implies (4). Property (5) and the property described in Lemma 5.7 are equivalent, merely written in different notation, and thus (5) and (1) are equivalent. It remains to prove $1 \Rightarrow 2 \Rightarrow 3$ and $4 \Rightarrow 5$.

$(1 \Rightarrow 2)$ Fix Polish spaces $E$ and $F, \bar{\mu}=\mu(d x) K_{x}^{\mu}(d y) \in \mathcal{P}(E \times F), f \in B(E \times F)$, and $g \in B(E)$ satisfying $\rho_{\mu}(g) \leq 0$. Suppose also

$$
\mu\left\{x \in E: \rho_{K_{x}^{\mu}}(f(x, \cdot)) \leq g(x)\right\}=1 .
$$

Find an $E \times F$-valued random variable $(X, Y)$ with law $\bar{\mu}$, and note that

$$
\rho(f(X, Y) \mid X)=\rho_{K_{X}^{\mu}}(f(X, \cdot)) \leq g(X), \text { a.s. }
$$

Acceptance consistency and monotonicity of $\rho$ yield

$$
\rho_{\bar{\mu}}(f)=\rho(f(X, Y)) \leq \rho(\rho(f(X, Y) \mid X)) \leq \rho(g(X))=\rho_{\mu}(g) \leq 0 .
$$

(2 $\Rightarrow 3$ ) Fix Polish spaces $E$ and $F, \bar{\mu}=\mu(d x) K_{x}^{\mu}(d y) \in \mathcal{P}(E \times F)$, and $f \in B(E \times F)$. Define $g \in B(E)$ by $g(x)=\rho_{K_{x}^{\mu}}(f(x, \cdot))$. Then trivially

$$
\mu\left\{x \in E: \rho_{K_{x}^{\mu}}\left(f(x, \cdot)-\rho_{\mu}(g)\right) \leq g(x)-\rho_{\mu}(g)\right\}=1 .
$$

Since $\rho_{\mu}\left(g-\rho_{\mu}(g)\right)=0$, property (2) implies

$$
\rho_{\bar{\mu}}\left(f-\rho_{\mu}(g)\right) \leq 0 .
$$

Rearrange this to get $\rho_{\bar{\mu}}(f) \leq \rho_{\mu}(g)$, as desired.

(4 $\Rightarrow$ 5) Fix Polish spaces $E$ and $F, \mu_{1} \in \mathcal{P}(E), \mu_{2} \in \mathcal{P}(F)$, and $f \in B(E \times F)$. Define $g \in B(E)$ by $g(x)=$ $\rho_{\mu_{2}}(f(x, \cdot))$. Then trivially

$$
\mu_{1}\left\{x \in E: \rho_{\mu_{2}}\left(f(x, \cdot)-\rho_{\mu}(g)\right) \leq g(x)-\rho_{\mu_{1}}(g)\right\}=1 .
$$

Since $\rho_{\mu_{1}}\left(g-\rho_{\mu_{1}}(g)\right)=0$, property (4) implies

$$
\rho_{\mu_{1} \times \mu_{2}}\left(f-\rho_{\mu_{1}}(g)\right) \leq 0 .
$$

Rearrange this to get $\rho_{\mu_{1} \times \mu_{2}}(f) \leq \rho_{\mu_{1}}(g)$, as desired.

This alternative description of acceptance consistency will serve us especially well when addressing additivity. For now, we will use it in establishing the connection between acceptance consistency and shift-convexity.

Proposition 5.9. A law-invariant risk measure is acceptance consistent if and only if its measure acceptance set is shift-convex. 
Proof. Let $\rho$ be a law-invariant risk measure with measure acceptance set $\mathcal{A}$. First, assume $\rho$ is acceptance consistent. Fix $\mu \in \mathcal{A}, M>0$, and a measurable map $\mathbb{R} \ni x \mapsto K_{x} \in \mathcal{A} \cap \mathcal{P}[-M, M]$. Set $\bar{\mu}=\mu(d x) K_{x}(d y-x)$. Letting $\lambda$ denote Lebesgue measure on [0,1], we may find (e.g. by [24, Theorem 5.10]) a measurable function $f: \mathbb{R} \times[0,1] \rightarrow \mathbb{R}$ such that, if $\hat{f}(x, y):=(x, f(x, y))$, then

$$
\bar{\mu}=(\mu \times \lambda) \circ \hat{f}^{-1} .
$$

Now set $g(x)=x$. Since $\lambda \circ f(x, \cdot)^{-1}=K_{x}(\cdot-x)$, we have

$$
\lambda \circ[f(x, \cdot)-g(x)]^{-1}=K_{x} \in \mathcal{A} \cap \mathcal{P}[-M, M],
$$

for each $x$. Thus

$$
\mu\left\{x \in \mathbb{R}: \rho_{\lambda}(f(x, \cdot)) \leq g(x)\right\}=\mu\left\{x \in \mathbb{R}: \lambda \circ[f(x, \cdot)-g(x)]^{-1} \in \mathcal{A}\right\}=1 .
$$

Note that since $\mu$ has compact support and $K_{x} \in \mathcal{P}[-M, M]$ for all $x$, it follows that $f$ is essentially bounded with respect to $\mu \times \lambda$. Since also $\mu \circ g^{-1}=\mu \in \mathcal{A}$, i.e., $\rho_{\mu}(g) \leq 0$, acceptance consistency (Proposition 5.8(2)) implies that $\rho_{\mu \times \lambda}(f) \leq 0$. In other words, $(\mu \times \lambda) \circ f^{-1} \in \mathcal{A}$. But this completes the proof of shift-convexity, since

$$
(\mu \times \lambda) \circ f^{-1}=\int_{\mathbb{R}} \mu(d x) K_{x}(\cdot-x) .
$$

Conversely, assume now that $\rho$ is shift-convex. Let $E$ and $F$ be Polish spaces, and fix $\mu_{1} \in \mathcal{P}(E), \mu_{2} \in \mathcal{P}(F)$, $f \in B(E \times F)$, and $g \in B(E)$ with $\rho_{\mu_{1}}(g) \leq 0$. Suppose also that

$$
\mu_{1}\left\{x \in E: \rho_{\mu_{2}}(f(x, \cdot)) \leq g(x)\right\}=1,
$$

or equivalently that

$$
\mu_{1}\left\{x \in E: \mu_{2} \circ[f(x, \cdot)-g(x)]^{-1} \in \mathcal{A}\right\}=1,
$$

In light of Proposition 5.8(4), we must check that $\rho_{\mu_{1} \times \mu_{2}}(f) \leq 0$, or equivalently that $\left(\mu_{1} \times \mu_{2}\right) \circ f^{-1} \in \mathcal{A}$. Set $v:=\mu_{1} \circ \mathrm{g}^{-1}$, and note that $v \in \mathcal{A}$. For $x \in \mathbb{R}$, define also

$$
K_{x}:= \begin{cases}\mu_{2} \circ[f(x, \cdot)-g(x)]^{-1} & \text { if } \mu_{2} \circ[f(x, \cdot)-g(x)]^{-1} \in \mathcal{A} \\ \delta_{0} & \text { otherwise. }\end{cases}
$$

(The choice of $\delta_{0}$ is arbitrary, and any other element of $\mathcal{A}$ would do.) Then $K_{x} \in \mathcal{A}$ for each $x$, and shiftconvexity implies

$$
\int_{\mathbb{R}} v(d x) K_{x}(\cdot-x) \in \mathcal{A} .
$$

But in fact $\int_{\mathbb{R}} v(d x) K_{x}(\cdot-x)$ is equal to $\left(\mu_{1} \times \mu_{2}\right) \circ f^{-1}$, since for $\phi \in B(\mathbb{R})$ we have

$$
\begin{aligned}
\int_{\mathbb{R}} v(d x) \int_{\mathbb{R}} K_{x}(d y-x) \phi(y) & =\int_{\mathbb{R}} v(d x) \int_{\mathbb{R}} K_{x}(d y) \phi(x+y) \\
& =\int_{E} \mu_{1}(d x) \int_{F} \mu_{2}(d y) \phi(g(x)+f(x, y)-g(x)) \\
& =\int_{E \times F} \phi \circ f d\left(\mu_{1} \times \mu_{2}\right) .
\end{aligned}
$$

Finally, before we turn to the proof of Theorem 5.5, we compute a penalty function for the risk measure $X \mapsto$ $\rho(\rho(X \mid \mathcal{G}))$, under no time consistency assumptions. This is related to some results in [1] and [10], but different in the sense that our conditional penalty functions are defined as pointwise suprema as opposed to essential suprema. Our result likely follows from the recent [5, Lemma 2.8], which appeared after the first version of this paper. 
Proposition 5.10. Let $\rho$ be a law invariant risk measure with induced divergence $\alpha$. Let $E$ and $F$ be Polish spaces, and let $\bar{\mu}=\mu(d x) K_{x}^{\mu}(d y) \in \mathcal{P}(E \times F)$. Let $f \in B(E \times F)$, and let $X$ denote the identity map on $E$. Then

$$
\rho_{\mu}\left(\left.\rho_{K_{x}^{\mu}}(f(x, \cdot))\right|_{x=X}\right)=\sup _{\bar{v}=v(d x) K_{x}^{\nu}(d y) \in \mathcal{P}(E \times F)}\left\{\int_{E \times f} f d \bar{\nu}-\int_{F} \alpha\left(K_{x}^{v} \mid K_{x}^{\mu}\right) v(d x)-\alpha(v \mid \mu)\right\} .
$$

Proof. We first compute

$$
\begin{aligned}
\rho_{\mu} & \left(\left.\rho_{K_{x}^{\mu}}(f(x, \cdot))\right|_{x=X}\right) \\
& =\sup _{v \in \mathcal{P}(E)}\left\{\int_{E} \rho_{K_{x}^{\mu}}(f(x, \cdot)) v(d x)-\alpha(v \mid \mu)\right\} \\
& =\sup _{v \in \mathcal{P}(E)}\left\{\int_{E} \sup _{\eta \in \mathcal{P}(F)}\left(\int_{F} f(x, y) \eta(d y)-\alpha\left(\eta \mid K_{x}^{\mu}\right)\right) v(d x)-\alpha(v \mid \mu)\right\} .
\end{aligned}
$$

Complete the proof by using a well known measurable selection argument [9, Proposition 7.50] to deduce

$$
\begin{aligned}
& \int_{E} \sup _{\eta \in \mathcal{P}(F)}\left(\int_{F} f(x, y) \eta(d y)-\alpha\left(\eta \mid K_{x}^{\mu}\right)\right) v(d x) \\
& \quad=\sup _{K}\left(\int_{E} \int_{F} f(x, y) K_{x}(d y) v(d x)-\int_{F} \alpha\left(K_{x} \mid K_{x}^{\mu}\right) v(d x,)\right),
\end{aligned}
$$

where the supremum is over all kernels from $E$ to $F$.

We have not shown that the penalty function identified in Proposition 5.10 is the minimal one, as it is not clear if it is lower semicontinuous or convex. This is the last lemma we need, and we defer its highly technical proof to Appendix A. It is here that we use the assumption that $\alpha$ is a simplified divergence. For closely related results, see [1, Lemma 4] and [5, Theorem 2.10]; the latter notably imposes the same restriction on $\alpha$ being simplified.

Lemma 5.11. Let $\rho$ be a law invariant risk measure with induced divergence $\alpha$, which we assume is simplified. Let $E$ and $F$ be Polish spaces, with $\bar{v}=v(d x) K_{x}^{v}(d y) \in \mathcal{P}(E \times F)$. Let $K$ be any kernel from $E$ to $F$. Then

$$
\int_{E} v(d x) \alpha\left(K_{x}^{v} \mid K_{x}\right)=\sup _{f \in B(E \times F)}\left\{\int f d \bar{v}-\int v(d x) \rho_{K_{x}}(f(x, \cdot))\right\} .
$$

\subsection{Proof of Theorem 5.5}

We saw in Proposition 5.9 that acceptance consistency and shift-convexity are equivalent. We will prove that acceptance consistency implies superadditivity and that partial superadditivity implies acceptance consistency. This is enough, since clearly superadditivity implies partial superadditivity. Fix throughout two Polish spaces $E$ and $F$ and a function $f \in B(E \times F)$. 
First assume $\rho$ is partially superadditive. Fix $\bar{\mu}=\mu_{1} \times \mu_{2} \in \mathcal{P}(E \times F)$. Use Proposition 5.10 followed by partial superadditivity to get

$$
\begin{aligned}
\rho_{\mu}\left(\left.\rho_{\mu_{2}}(f(x, Y))\right|_{x=X}\right) & =\sup _{v(d x) K_{x}^{v}(d y) \in \mathcal{P}(E \times F)}\left\{\int_{E} \int_{F} f(x, y) K_{x}^{v}(d y) v(d x)-\int_{F} \alpha\left(K_{x}^{v} \mid \mu_{2}\right) v(d x)-\alpha\left(v \mid \mu_{1}\right)\right\} \\
& \geq \sup _{v(d x) K_{x}^{v}(d y) \in \mathcal{P}(E \times F)}\left\{\int_{E} \int_{F} f(x, y) K_{x}^{v}(d y) v(d x)-\alpha\left(v(d x) K_{x}^{v}(d y) \mid \bar{\mu}\right)\right\} \\
& =\rho_{\bar{\mu}}(f) .
\end{aligned}
$$

Conclude from Proposition 5.8(5) that $\rho$ is acceptance consistent.

Now suppose $\rho$ is acceptance consistent. Let $\bar{\mu}=\mu(d x) K_{x}^{\mu}(d y) \in \mathcal{P}(E \times F)$. Use Proposition 5.10 followed by Proposition 5.8(3) to get

$$
\begin{aligned}
\sup _{v(d x) K_{x}^{\nu}(d y) \in \mathcal{P}(E \times F)}\left\{\int_{E} \int_{F} f(x, y) K_{x}^{v}(d y) v(d x)-\int_{F} \alpha\left(K_{x}^{v} \mid K_{x}^{\mu}\right) v(d x)-\alpha(v \mid \mu)\right\} & =\rho_{\mu}\left(\left.\rho_{K_{x}^{\mu}}(f(x, \cdot))\right|_{x=X}\right) \\
& \geq \rho_{\bar{\mu}}(f)
\end{aligned}
$$

On the other hand, using Lemma 5.11 and the definition of $\alpha$, we get

$$
\begin{aligned}
\int v(d x) \alpha\left(K_{x}^{v} \mid K_{x}^{\mu}\right)+\alpha(v \mid \mu) & =\sup _{f \in B(E \times F), g \in B(E)}\left\{\int f d \bar{v}-\int v(d x) \rho_{K_{x}^{\mu}}(f(x, \cdot))+\int g d v-\rho_{\mu}(g)\right\} \\
& =\sup _{f \in B(E \times F), g \in B(E)}\left\{\int(g(x)+f(x, y)) \bar{v}(d x, d y)-\rho_{\mu}\left(g+\rho_{K_{x}^{\mu}}(f(x, \cdot))\right)\right\} \\
& =\sup _{f \in B(E \times F)}\left\{\int f d \bar{v}-\rho_{\mu}\left(\left.\rho_{K_{x}^{\mu}}(f(x, \cdot))\right|_{x=X}\right)\right\} .
\end{aligned}
$$

Indeed, in the second line we replaced $g(x)$ by $g(x)+\rho_{K_{x}^{\mu}}(f(x, \cdot))$, and in the final step we replaced $f$ by $f-g$. This shows that the function of $v(d x) K_{x}^{v}(d y) \in \mathcal{P}(E \times F)$ given by

$$
\int_{F} \alpha\left(K_{x}^{v} \mid K_{x}^{\mu}\right) v(d x)+\alpha(v \mid \mu)
$$

is precisely the minimal penalty function of the risk measure given by (5.1). Since $\bar{v} \mapsto \alpha(\bar{v} \mid \bar{\mu})$ is the minimal penalty function of $\rho_{\bar{\mu}}$ (see Theorem 2.2), it follows from the order-reversing property of convex conjugation that $\alpha(\cdot \mid \bar{\mu})$ dominates the minimal penalty function of the risk measure given by (5.1). That is,

$$
\alpha\left(v(d x) K_{x}^{v}(d y) \mid \bar{\mu}\right) \geq \int_{F} \alpha\left(K_{x}^{v} \mid K_{x}^{\mu}\right) v(d x)+\alpha(v \mid \mu),
$$

for every $v(d x) K_{x}^{v}(d y) \in \mathcal{P}(E \times F)$.

\subsection{Weak time consistency}

A related notion of time consistency was studied by Weber in [38]. Namely, we say a law invariant risk measure $\rho$ is weakly acceptance consistent if $\rho(X \mid \mathcal{G}) \leq 0$ a.s. implies $\rho(X) \leq 0$, for every $X \in L^{\infty}$ and every $\sigma$-field $\mathcal{G} \subset \mathcal{F}$. Similarly, $\rho$ is weakly rejection consistent if $\rho(X \mid \mathcal{G})>0$ a.s. implies $\rho(X)>0$. The following result, due in large part to Weber [38], characterizes weak time consistency in terms of measure acceptance sets as well as divergences. Let us say that a set $\mathcal{A} \subset \mathcal{P}(\mathbb{R})$ is locally measure convex if for each $M>0$ and each $Q \in \mathcal{P}(\mathcal{A} \cap \mathcal{P}[-M, M])$ the mean measure $\int_{\mathcal{A} \cap \mathcal{P}[-M, M]} Q(\mathrm{dm}) m$ is in $\mathcal{A}$. 
Theorem 5.12. Suppose $\alpha$ is a simplified divergence induced by a law invariant risk measure $\rho$ with acceptance set $\mathcal{A}$. The following are equivalent:

(1) $\rho$ is weakly acceptance consistent.

(2) $\mathcal{A}$ is locally measure convex.

(3) For Polish spaces $E$ and $F$, and measures $\mu(d x) K_{x}^{\mu}(d y)$ and $v(d x) K_{x}^{v}(d y)$ in $\mathcal{P}(E \times F)$, we have

$$
\alpha\left(v(d x) K_{x}^{v}(d y) \mid \mu(d x) K_{x}^{\mu}(d y)\right) \geq \int v(d x) \alpha\left(K_{x}^{v} \mid K_{x}^{\mu}\right) .
$$

(4) For Polish spaces $E$ and $F$, and measures $\mu_{1} \times \mu_{2}$ and $v(d x) K_{x}^{v}(d y)$ in $\mathcal{P}(E \times F)$, we have

$$
\alpha\left(v(d x) K_{x}^{v}(d y) \mid \mu_{1} \times \mu_{2}\right) \geq \int v(d x) \alpha\left(K_{x}^{v} \mid \mu_{2}\right) .
$$

Similarly, the same equivalences hold when "acceptance" is changed to "rejection", $\geq$ is is changed to $\leq$, and $\mathcal{A}$ is changed to $\mathcal{A}^{c}$. The equivalence of (1) and (2) holds without the assumption that $\alpha$ is simplified.

Proof. The implication (1) $\Leftrightarrow(2)$ in the following was first noticed by Weber [38], and the rest is proven along the same lines as Theorem 5.5, but we provide a sketch: Suppose first that (1) holds. Fix Polish spaces $E$ and $F$ and measures $\mu(d x) K_{x}^{\mu}(d y)$ and $v(d x) K_{x}^{\nu}(d y)$ in $\mathcal{P}(E \times F)$. It is easy to see (similar to Proposition 5.8) that weak acceptance consistency is equivalent to the following: for $f \in B(E \times F)$,

$$
\mu\left\{\rho_{K_{x}^{\mu}}(f(x, \cdot)) \leq 0\right\}=1 \quad \Rightarrow \quad \rho_{\bar{\mu}}(f) \leq 0 .
$$

Thus, by Lemma 5.11,

$$
\begin{aligned}
\int v(d x) \alpha\left(K_{x}^{v} \mid K_{x}^{\mu}\right) & =\sup _{f \in B(E \times F)}\left\{\int f d \bar{v}-\int v(d x) \rho_{K_{x}^{\mu}}(f(x, \cdot))\right\} \\
& =\sup \left\{\int f d \bar{v}: f \in B(E \times F), \mu\left\{\rho_{K_{x}^{\mu}}(f(x, \cdot)) \leq 0\right\}=1\right\} \\
& \leq \sup \left\{\int f d \bar{v}: f \in B(E \times F), \rho_{\bar{\mu}}(f) \leq 0\right\} \\
& =\alpha(\bar{v} \mid \bar{\mu}) .
\end{aligned}
$$

This proves (1) $\Rightarrow$ (3). Since clearly (3) implies (4), let us finally show that (4) implies (1). Fix Polish spaces $E$ and $F$ and $\mu_{1} \times \mu_{2} \in \mathcal{P}(E \times F)$. As in the proof of Theorem 5.5, the inequality of (4), combined with Lemma 5.11 and the order-reversing property of convex conjugation, implies the set inclusion

$$
\left\{f \in B(E \times F): \rho_{\mu_{1} \times \mu_{2}}(f) \leq 0\right\} \supset\left\{f \in B(E \times F): \mu_{1}\left\{\rho_{\mu_{2}}(f(x, \cdot)) \leq 0\right\}=1\right\} .
$$

Again, it is easy to see (similar to Proposition 5.8) this implies weak acceptance consistency.

\subsection{Some comments on measure convexity and shift-convexity}

For a measure acceptance set $\mathcal{A}$, local measure convexity is equivalent to (ordinary) convexity. Indeed, the set $\mathcal{A} \cap \mathcal{P}[-M, M]$ is weakly closed for each $M>0$, which can be proven easily using the Fatou property 2.1 and the Skorohod representation for weak convergence. It is well known that closed convex subsets of $\mathcal{P}[-M, M]$ are measure convex, e.g. [39, Corollary 1.2.4]. The same equivalence may not hold for the complement $\mathcal{A}^{c}$, which is not closed.

The above fact is rather remarkable, in the sense that the property of local measure convexity of $\mathcal{A}$, which a priori involves infinite convex combinations, can be reduced to checking that the convex combination of 
any pair of points remains in the set. It is not clear if such a reduction is possible for the notion of shiftconvexity, and a key obstruction is that the probability measure $\mu$ in Definition 5.4(2), which governs the convex combination, must itself belong to the set $\mathcal{A}$.

It is true, however, that if $\mathcal{A}=\left\{P \circ X^{-1}: X \in L^{\infty}, \rho(X) \leq 0\right\}$ is the measure acceptance set of a law invariant risk measure $\rho$, then to check shift-convexity of $\mathcal{A}$ it suffices to check Definition 5.4(2) only for finitely supported measures $\mu \in \mathcal{A}$ and $K_{x} \in \mathcal{A}$ for each $x$. This follows from two observations. First, given $\mu \in \mathcal{A}$, a measurable map $\mathbb{R} \ni x \mapsto K_{x} \in \mathcal{A} \cap P([-M, M])$ can be approximated (weakly) pointwise by simple (i.e., finite-range) functions such that the set of continuity points of $x \mapsto K_{x}$ has full $\mu$-measure. Second, we may approximate $\mu$ and $K_{x}$ weakly by $\mu \circ \pi_{n}^{-1}$ and $K_{x} \circ \pi_{n}^{-1}$, where $\pi_{n}(x):=\lfloor n x\rfloor / n$ for $x \in \mathbb{R}$, noting that the monotonicity of $\rho$ ensures that $v \circ \pi_{n}^{-1} \in \mathcal{A}$ whenever $v \in \mathcal{A}$.

\section{Further properties of divergences}

Divergences are actually uniquely determined by their values for finite spaces $E$, as is formalized in the following proposition. Building on the characterization of relative entropy in Corollary 5.6 below, we could derive an even simpler characterization akin to those surveyed by Csiszár [11], but this would lead us too far astray.

Proposition 6.1. Suppose $\alpha$ is a simplified divergence. For any Polish space $E$ and any $\mu, v \in \mathcal{P}(E)$, we have

$$
\alpha(v \mid \mu)=\sup \left\{\alpha\left(\nu \circ T^{-1} \mid \mu \circ T^{-1}\right): T: E \rightarrow F \text { measurable, } F \text { finite }\right\} .
$$

Proof. The inequality $\geq$ follows immediately from the definition of a divergence. To prove the reverse inequality, note that it holds trivially if $E$ is finite. Generally, by Borel isomorphism (see [25, Theorem 15.6]), there exists a measurable function $S: E \rightarrow[0,1]$ with measurable inverse. Suppose we can prove that

$$
\alpha(v \mid \mu)=\sup \left\{\alpha\left(v \circ T^{-1} \mid \mu \circ T^{-1}\right): T:[0,1] \rightarrow F \text { measurable, } F \text { finite }\right\} .
$$

for all $\mu, v \in \mathcal{P}([0,1])$. Then, if $\mu, v \in \mathcal{P}(E)$, we use Proposition 2.6 to conclude

$$
\begin{aligned}
\alpha(v \mid \mu) & =\alpha\left(v \circ S^{-1} \mid \mu \circ S^{-1}\right) \\
& =\sup \left\{\alpha\left(v \circ(T \circ S)^{-1} \mid \mu \circ(T \circ S)^{-1}\right): T:[0,1] \rightarrow F \text { measurable, } F \text { finite }\right\} \\
& =\sup \left\{\alpha\left(v \circ T^{-1} \mid \mu \circ T^{-1}\right): T: E \rightarrow F \text { measurable, } F \text { finite }\right\} .
\end{aligned}
$$

Indeed, this is true because every measurable map $T: E \rightarrow F$ can be written as $T^{\prime} \circ S$, where $T^{\prime}=T \circ S^{-1}$. Hence, we need only to prove (6.1).

Since $[0,1]$ is compact, for each $n$ we may find a measurable map $T_{n}:[0,1] \rightarrow[0,1]$ with finite range such that $\left|x-T_{n}(x)\right| \leq 1 / n$ for all $x \in[0,1]$. Then $T_{n}$ converges uniformly to the identity. Since $\alpha$ is simplified, for a given $\epsilon>0$ we may find a continuous function $f$ on $[0,1]$ such that

$$
\alpha(v \mid \mu) \leq \epsilon+\int f d v-\rho_{\mu}(f) .
$$

Since $\rho_{\mu}$ is continuous in the supremum norm, and since $f \circ T_{n} \rightarrow f$ uniformly, we conclude that $\rho_{\mu}(f)=$ $\lim _{n} \rho_{\mu}\left(f \circ T_{n}\right)$. Thus

$$
\begin{aligned}
\alpha(v \mid \mu) & \leq \epsilon+\lim _{n \rightarrow \infty}\left(\int f \circ T_{n} d v-\rho_{\mu}\left(f \circ T_{n}\right)\right) \\
& =\epsilon+\lim _{n \rightarrow \infty}\left(\int f d v \circ T_{n}^{-1}-\rho_{\mu \circ T_{n}^{-1}}(f)\right) \\
& \leq \epsilon+\liminf _{n \rightarrow \infty} \alpha\left(v \circ T_{n}^{-1} \mid \mu \circ T_{n}^{-1}\right) .
\end{aligned}
$$

This is enough to complete the proof. 
Finally, let us mention a result of potential relevance in mathematical statistics, namely that sufficient statistics always attain equality in the inequality $\alpha\left(\nu \circ T^{-1} \mid \mu \circ T^{-1}\right) \leq \alpha(v \mid \mu)$. The foundational paper [26] of Kullback and Leibler proved this result for relative entropy, and Liese and Vajda [32, Theorem 14] treat the case of $f$ divergences.

Proposition 6.2. Let $E$ be a Polish space and $\mu, v \in \mathcal{P}(E)$ with $v \ll \mu$. Suppose a measurable map $T: E \rightarrow F$ is sufficient for $\{\mu, v\}$, meaning that $d v / d \mu$ is T-measurable. Then, for any divergence $\alpha$, we have $\alpha\left(v \circ T^{-1} \mid \mu \circ\right.$ $\left.T^{-1}\right)=\alpha(v \mid \mu)$. In particular, this holds if $T$ is a bijection with measurable inverse.

Proof. By definition of a divergence, $\alpha\left(v \circ T^{-1} \mid \mu \circ T^{-1}\right) \leq \alpha(v \mid \mu)$, so we must only prove the reverse inequality. By assumption, $d v / d \mu=\varphi \circ T$ for some measurable $\varphi: F \rightarrow \mathbb{R}$. As we are working on Polish spaces, we may find a kernel $K$ from $F$ to $E$ such that $\mu(d x) \delta_{T(x)}(d y)=\mu \circ T^{-1}(d y) K_{y}(d x)$. We claim that $\mu=\left(\mu \circ T^{-1}\right) K$ and $v=\left(v \circ T^{-1}\right) K$. Once this is proven, the proof is completed by applying Property (3) of Theorem 2.7. To prove the claim, note first that $\mu=\left(\mu \circ T^{-1}\right) K$ is immediate. To prove $v=\left(v \circ T^{-1}\right) K$, fix a Borel set $A \subset E$ and compute

$$
\begin{aligned}
\left(v \circ T^{-1}\right) K(A) & =\int_{F} K_{y}(A)\left(v \circ T^{-1}\right)(d y)=\int_{E} K_{T(x)}(A) v(d x) \\
& =\int_{E} K_{T(x)}(A) \varphi(T(x)) \mu(d x)=\iint_{F} \int_{E} 1_{A}(x) \varphi(y) K_{y}(d x)\left(\mu \circ T^{-1}\right)(d y) \\
& =\int_{E} 1_{A}(x) \varphi(T(x)) \mu(d x)=v(A) .
\end{aligned}
$$

Remark 6.3. Proposition 6.2 raises a natural question: Does the converse hold? That is, does the equality $\alpha\left(v \circ T^{-1} \mid \mu \circ T^{-1}\right)=\alpha(v \mid \mu)$ imply that $T$ is sufficient for $(\mu, v)$ ? This does not hold for all divergences, but it does when $\alpha$ is relative entropy, as was observed first by Kullback and Leibler [26]. Liese and Vajda [32] show that this converse holds for many (but not all) $f$-divergences. This characterization leads to useful tests for sufficiency, as is explained in both of these papers [26, 32].

\section{Examples}

Before we discuss some common law invariant risk measures, recall that our sign convention is not the usual one. Namely, $\rho$ is increasing, not decreasing. More precisely, if $\rho$ is a risk measure according to our definition, the map $X \mapsto \rho(-X)$ is what is more often called a risk measure, as in [18].

\subsection{Shortfall risk measures}

Shortfall risk measures, introduced by Föllmer and Schied [17], are of the form

$$
\rho(X)=\inf \{c \in \mathbb{R}: \mathbb{E}[\ell(X-c)] \leq 1\},
$$

where $\ell$ is a loss function, defined as follows:

Definition 7.1. A loss function is a convex and nondecreasing function $\ell: \mathbb{R} \rightarrow \mathbb{R}$ satisfying $\ell(0)=1<\ell(x)$ for all $x>0$. 
Of course, the induced family of risk measures is

$$
\rho_{\mu}(f)=\inf \left\{c \in \mathbb{R}: \int_{E} \ell(f(x)-c) \mu(d x) \leq 1\right\} .
$$

Note that by continuity of $\ell$ and monotone convergence, the infimum is always attained. In particular,

$$
\int_{E} \ell\left(f(x)-\rho_{\mu}(f)\right) \mu(d x) \leq 1
$$

According to the [18, Theorem 4.115] the induced divergence is

$$
\alpha(v \mid \mu)=\inf _{t>0} \frac{1}{t}\left(1+\int_{E} \ell^{\star}\left(t \frac{d v}{d \mu}\right) d \mu\right), \text { for } v \ll \mu,
$$

where $\ell^{\star}(x)=\sup _{y \in \mathbb{R}}(x y-\ell(y))$ is the convex conjugate. It is known that shortfall risk measures are Lebesgue continuous in the sense of Definition 3.3 [18, Proposition 4.113 and Exercise 4.2.2]. Hence, by Theorem 3.4, $\alpha$ is jointly weakly lower semicontinuous and simplified.

Let us now describe some examples of shortfall risk measures which are acceptance consistent. We say that a positive function $\ell: \mathbb{R} \rightarrow(0, \infty)$ is log-subadditive (resp. log-superadditive) if $\ell(x+y) \leq \ell(x) \ell(y)$ (resp. $\geq$ ) for all $x, y \in \mathbb{R} .^{3}$

Proposition 7.2. Let $\ell$ be a loss function and $\alpha$ the corresponding divergence defined in (7.2). If $\ell$ is $\log$ subadditive (resp. log-superadditive) then $\alpha$ is superadditive (resp. subadditive), or equivalently $\rho$ is acceptance consistent (resp. rejection consistent)

Proof. Assume $\ell$ is log-subadditive. With Theorem 5.5 in mind, we will show that the following set is shiftconvex:

$$
\mathcal{A}:=\left\{m \in \bigcup_{M>0} \mathcal{P}[-M, M]: \int \ell d m \leq 1\right\} .
$$

Fix $\mu \in \mathcal{A}, M>0$, and a measurable map $\mathbb{R} \ni x \mapsto K_{x} \in \mathcal{A} \cap \mathcal{P}[-M, M]$. Then $\int_{\mathbb{R}} \ell d K_{x} \leq 1$ for all $x$ and also $\int_{\mathbb{R}} \ell d \mu \leq 1$. Thus

$$
\begin{aligned}
\int_{\mathbb{R}} \mu(d x) \int_{\mathbb{R}} K_{x}(d y-x) \ell(y) & =\int_{\mathbb{R}} \mu(d x) \int_{\mathbb{R}} K_{x}(d y) \ell(y+x) \\
& \leq \int_{\mathbb{R}} \mu(d x) \ell(x) \int_{\mathbb{R}} K_{x}(d y) \ell(y) \\
& \leq 1,
\end{aligned}
$$

and it follows that $\int_{\mathbb{R}} \mu(d x) K_{x}(\cdot-x)$ is in $\mathcal{A}$.

Remark 7.3. It is difficult to construct interesting examples of log-subadditive functions, beyond the obvious case of $\ell(x)=e^{\eta x}$ for $\eta>0$. The function $\log \ell$ must be nondecreasing and subadditive on $\mathbb{R}$ and equal to zero at zero, and moreover the exponential of this function must be convex. The only other examples we found are all of the restrictive form $\ell(x)=e^{F(x)}$ for nondecreasing functions $F$ with $F^{\prime}(0)>0$ and with at most linear growth, i.e., $F(x) \leq c_{1} x$ for all $x \geq 0$ and $F(x) \geq c_{2} x$ for $x \leq 0$, for $c_{1}, c_{2}>0$.

3 Note that a loss function $\ell$ satisfying $\ell(x)=0$ for some $x$ cannot be log-subadditivity, as this would imply $\ell(y) \leq \ell(y-x) \ell(x)=0$ for all $y$. This is why we restrict here to positive functions. 
A shortfall risk measure can still be acceptance consistent even if its loss function is not log-subadditive or even positive. An interesting family of examples is the family of expectiles (see [6] and references therein). ${ }^{4}$ If $\ell(x)=1+b x^{+}-c x^{-}$, where $b \geq c>0$, then $\ell^{\star}(x)=-1$ for $c \leq x \leq b$ and $\ell^{*}(x)=\infty$ otherwise, and $\alpha$ in (7.2) becomes

$$
\alpha(v \mid \mu)= \begin{cases}0 & \text { if } \operatorname{esssup}^{\mu} \frac{d v}{d \mu} \leq \frac{b}{c} \operatorname{essinf}^{\mu} \frac{d v}{d \mu}, \\ \infty & \text { otherwise }\end{cases}
$$

where ess $\sup ^{\mu}$ and ess inf ${ }^{\mu}$ to denote essential supremum and infimum under $\mu$.

Proposition 7.4. Define $\alpha$ as in (7.4). Then $\alpha$ is superadditive, and $\rho$ is acceptance consistent.

Proof. With Theorem 5.5 in mind, we will show that the set $\mathcal{A}$ of (7.3) is shift-convex, now with $\ell(x)=1+b x^{+}-$ $c x^{-}=1+(b-c) x^{+}+c x$. Fix $\mu \in \mathcal{A}, M>0$, and a measurable map $\mathbb{R} \ni x \mapsto K_{x} \in \mathcal{A} \cap \mathcal{P}[-M, M]$. Then

$$
\int_{\mathbb{R}} \mu(d x)\left(1+(b-c) x^{+}+c x\right) \leq 1, \quad \sup _{x \in \mathbb{R}} \int_{\mathbb{R}} K_{x}(d y)\left(1+(b-c) y^{+}+c y\right) \leq 1 .
$$

Hence, using subadditivity of $x \mapsto x^{+}$,

$$
\begin{aligned}
\int_{\mathbb{R}} \mu(d x) \int_{\mathbb{R}} K_{x}(d y-x) \ell(y) & =\int_{\mathbb{R}} \mu(d x) \int_{\mathbb{R}} K_{x}(d y)\left(1+(b-c)(x+y)^{+}+c(x+y)\right) \\
& \leq \int_{\mathbb{R}} \mu(d x)\left((b-c) x^{+}+c x+\int_{\mathbb{R}} K_{x}(d y)\left(1+(b-c) y^{+}+c y\right)\right) \\
& \leq \int_{\mathbb{R}} \mu(d x)\left(1+(b-c) x^{+}+c x\right) \\
& \leq 1,
\end{aligned}
$$

which shows that $\int_{\mathbb{R}} \mu(d x) K_{x}(\cdot-x)$ is in $\mathcal{A}$.

\subsection{Optimized certainty equivalent}

An optimized certainty equivalent, as introduced by Ben-Tal and Teboulle [7, 8], is of the form

$$
\rho(X):=\inf _{m \in \mathbb{R}}(\mathbb{E}[\phi(m+X)]-m),
$$

where $\phi: \mathbb{R} \rightarrow \mathbb{R}$ is convex and nondecreasing, with $\phi^{\star}(1)=\sup _{x \in \mathbb{R}}(x-\phi(x))=0$. Of course, the induced family of risk measures is

$$
\rho_{\mu}(f):=\inf _{m \in \mathbb{R}}\left(\int_{E} \phi(m+f(x)) \mu(d x)-m\right) .
$$

The corresponding divergence is the $\phi^{\star}$-divergence [31, 32],

$$
\alpha(v \mid \mu)=\int \phi^{\star}\left(\frac{d v}{d \mu}\right) d \mu, \text { for } v \ll \mu .
$$

As we saw in the discussion preceding Proposition 4.1, an optimized certainty equivalent always satisfies the concavity condition of Proposition 4.1, and this provides an alternative proof of the well known joint convexity of $\alpha$. It is also known that $\alpha$ is jointly weakly lower semicontinuous, which we confirm using Theorem 3.4 before addressing time consistency and additivity properties.

4 Many thanks to an anonymous referee for pointing out this example. 
Proposition 7.5. Every optimized certainty equivalent is weakly lower semicontinuous. In particular, $\alpha$ is simplified.

Proof. The second claim follows from the first by Theorem 3.4. According to Theorem 3.4, it suffices to show Lebesgue continuity: If $X_{n}, X \in L^{\infty}$ with $X_{n} \downarrow X$ a.s., we must show that $\rho\left(X_{n}\right) \downarrow \rho(X)$. Let $\epsilon>0$, and find $m \in \mathbb{R}$ such that

$$
\mathbb{E}[\phi(m+X)]-m \leq \rho(X)+\epsilon .
$$

By monotone convergence, $\mathbb{E}\left[\phi\left(m+X_{n}\right)\right] \downarrow \mathbb{E}[\phi(m+X)]$. Thus, for sufficiently large $n$,

$$
\rho\left(X_{n}\right) \leq \mathbb{E}\left[\phi\left(m+X_{n}\right)\right]-m \leq \mathbb{E}[\phi(m+X)]-m+\epsilon \leq \rho(X)+2 \epsilon .
$$

Theorem 7.6. Suppose $\phi$ satisfies $y \phi^{\star}(x)+x \phi^{\star}(y) \leq \phi^{\star}(x y)$ (resp. $\left.\geq\right)$ for all $x, y \geq 0$. Then $\alpha$ is superadditive (resp. subadditive), or equivalently $\rho$ is acceptance consistent (resp. rejection consistent).

Proof. We treat the superadditive case, as the subadditive case is proven similarly. Suppose $E$ and $F$ are Polish spaces, and let $\bar{\mu}=\mu(d x) K_{x}^{\mu}(d y)$ and $\bar{v}=v(d x) K_{x}^{v}(d y)$ be probability measures on $E \times F$. Simply use the definition of $\alpha$ :

$$
\begin{aligned}
\alpha(\bar{v} \mid \bar{\mu}) & =\int_{E \times F} \phi^{\star}\left(\frac{d \bar{v}}{d \bar{\mu}}\right) d \bar{\mu} \\
& =\int_{E} \mu(d x) \int_{F} K_{x}^{\mu}(d y) \phi^{\star}\left(\frac{d v}{d \mu}(x) \frac{d K_{x}^{v}}{d K_{x}^{\mu}}(y)\right) \\
& \geq \int_{E} \mu(d x) \int_{F} K_{x}^{\mu}(d y)\left[\frac{d K_{x}^{v}}{d K_{x}^{\mu}}(y) \phi^{\star}\left(\frac{d v}{d \mu}(x)\right)+\frac{d v}{d \mu}(x) \phi^{\star}\left(\frac{d K_{x}^{v}}{d K_{x}^{\mu}}(y)\right)\right] \\
& =\int_{E} \phi^{\star}\left(\frac{d v}{d \mu}\right) d \mu+\int_{E} v(d x) \int_{F} K_{x}^{\mu}(d y) \phi^{\star}\left(\frac{d K_{x}^{v}}{d K_{x}^{\mu}}(y)\right) \\
& =\alpha(v \mid \mu)+\int_{E} v(d x) \alpha\left(K_{x}^{v} \mid K_{x}^{\mu}\right) .
\end{aligned}
$$

Remark 7.7. Of course, the relationship $\phi^{\star}(x y)=x \phi^{\star}(y)+y \phi^{\star}(x)$ is satisfied by $\phi^{\star}(x)=x \log x$, the conjugate of which (assuming $\phi^{\star}=+\infty$ on the negative half-line) is $\phi(x)=e^{x-1}$. More generally, suppose $\ell$ is a strictly increasing log-subadditive loss function. Then $\ell^{-1}(x y) \geq \ell^{-1}(x)+\ell^{-1}(y)$ for $x, y>0$, and so $\phi^{\star}(x):=x \ell^{-1}(x)$ satisfies $\phi^{\star}(x y) \geq x \phi^{\star}(y)+y \phi^{\star}(x)$. As was discussed in Remark 7.3, there are not many such functions.

\subsection{Coherent risk measures}

A risk measure is called coherent if $\rho(\lambda X)=\lambda \rho(X)$ for all $X \in L^{\infty}$ and $\lambda \geq 0$. A coherent law invariant risk measure admits a representation

$$
\rho(X)=\sup _{Q \in Q} \mathbb{E}^{Q}[X]
$$

where $\mathcal{Q} \subset \mathcal{P}_{P}(\Omega)$ is convex and closed with respect to total variation. Law invariance simply means that if $Q \in \mathcal{Q}$ and $Q^{\prime} \in \mathcal{P}_{P}(\Omega)$ have the same density law $P \circ(d Q / d P)^{-1}=P \circ\left(d Q^{\prime} / d P\right)^{-1}$, then $Q^{\prime}$ must also be in $Q$; indeed, this follows easily from the Kusuoka representation (see [23, 28]).

Coherence is easy to characterize in terms of divergences, because the minimal penalty function of a coherent risk measure must take values in $\{0, \infty\}$. Hence, the divergence $\alpha$ induced by a coherent risk measure 
must be of the form

$$
\alpha(v \mid \mu)= \begin{cases}0 & \text { if } \nu \in \mathcal{Q}[\mu] \\ \infty & \text { if } \nu \notin \mathcal{Q}[\mu],\end{cases}
$$

for each Polish space $E$ and each $\mu \in \mathcal{P}(E)$, where the set $Q[\mu] \subset \mathcal{P}(E)$ is convex and closed with respect to total variation. On the other hand, if we are given a function $\alpha$ of this form and we want $\alpha$ to be divergence in the sense of Definition 2.8, we must must have the following three properties, which correspond exactly to the defining properties (1-3) from Theorem 2.7:

1. $\mu \in \mathcal{Q}[\mu]$ for each Polish space $E$ and each $\mu \in \mathcal{P}(E)$,

2. $Q[\mu] \subset \mathcal{P}_{\mu}(E)$ for each Polish space $E$ and each $\mu \in \mathcal{P}(E)$,

3. $v K \in \mathcal{Q}[\mu K]$ for any Polish spaces $E$ and $F$, any $v, \mu \in \mathcal{P}(E)$, and any kernel $K$ from $E$ to $F$.

Let us call such a divergence a coherent divergence. One natural way to construct a coherent divergence $\alpha(\cdot \cdot \cdot)$ from an arbitrary divergence $\beta(\cdot \mid \cdot)$ is as follows: Fix $c>0$, and define $Q[\mu]=\{v: \beta(v \mid \mu) \leq c\}$.

A coherent divergence is simplified, in the sense of Definition 2.10, precisely when the set $Q[\mu]$ is weakly closed for every $\mu$. Joint weak lower semicontinuity in the sense of Definition 3.2 is clearly equivalent to the weak closedness of the graph $\operatorname{Gr}(\mathcal{Q})=\left\{(\nu, \mu) \in \mathcal{P}(E)^{2}: \nu \in \mathcal{Q}[\mu]\right\}$. Likewise, joint convexity is equivalent to convexity of the set $\operatorname{Gr}(Q)$.

Let us next address the question of when a simplified coherent divergence $\alpha$ is sub or superadditive. Using the form of Definition 5.3, we see that $\alpha$ is partially superadditive if and only if

$$
\mathcal{Q}\left[\mu_{1} \times \mu_{2}\right] \subset \mathcal{Q}\left[\mu_{1}, \mu_{2}\right],
$$

for every pair of Polish spaces $E_{1}$ and $E_{2}$ and every $\mu_{i} \in \mathcal{P}\left(E_{i}\right)$, where we define

$$
\mathcal{Q}\left[\mu_{1}, \mu_{2}\right]:=\left\{m(d x) K_{x}(d y) \in \mathcal{P}\left(E_{1} \times E_{2}\right): m \in \mathcal{Q}\left[\mu_{1}\right], K_{x} \in \mathcal{Q}\left[\mu_{2}\right] \text { for all } x\right\} .
$$

In other words, $Q$ is the set of joint laws in which the first marginal lies in $Q\left[\mu_{1}\right]$ and the conditional law of the second variable belongs almost surely to $Q\left[\mu_{2}\right]$. For this to hold for every pair $\mu_{1}, \mu_{2}$ is a very stringent requirement, and we were unable to clarify it any further. It holds in the extreme cases, when $Q[\mu]=\{\mu\}$ for all $\mu$ or $\mathrm{Q}[\mu]=\mathcal{P}_{\mu}(\Omega)$ for all $\mu$, as well as for expectiles, as treated in Proposition 7.4. Indeed, the choice of $\alpha$ in (7.4) is superadditive and corresponds to the sets

$$
\mathcal{Q}[\mu]=\left\{v \ll \mu: \operatorname{ess~sup}^{\mu} \frac{d v}{d \mu} \leq \frac{b}{c} \operatorname{essinf}^{\mu} \frac{d v}{d \mu}\right\} .
$$

\section{A Proof of Lemma 5.11}

Keep the notation of Lemma 5.11. For each $x \in E$ we have

$$
\alpha\left(K_{x}^{v} \mid K_{x}^{\mu}\right)=\sup _{f \in B(F)}\left(\int_{F} f d K_{x}^{v}-\rho_{K_{x}^{\mu}}(f)\right),
$$

and this clearly implies (recalling that $\bar{v}:=v(d x) K_{x}^{v}(d y)$ ) that

$$
\int_{E} v(d x) \alpha\left(K_{x}^{v} \mid K_{x}^{\mu}\right) \geq \sup _{f \in B(E \times F)}\left\{\int_{E \times F} f d \bar{v}-\int_{E} v(d x) \rho_{K_{x}^{\mu}}(f(x, \cdot))\right\} .
$$

The rest of the proof is devoted to establishing the trickier inequality:

$$
\int_{E} v(d x) \alpha\left(K_{x}^{v} \mid K_{x}^{\mu}\right) \leq \sup _{f \in B(E \times F)}\left\{\int_{E \times F} f d \bar{v}-\int_{E} v(d x) \rho_{K_{x}^{\mu}}(f(x, \cdot))\right\} .
$$


Proof for $F=[0,1]$. Assume first that $F=[0,1]$. Since $\alpha$ is simplified, for each $x \in E$ we have

$$
\alpha\left(K_{x}^{v} \mid K_{x}^{\mu}\right)=\sup _{f \in C([0,1])}\left(\int_{[0,1]} f d K_{x}^{v}-\rho_{K_{x}^{\mu}}(f)\right) .
$$

Now note that for each $f \in C([0,1])$, the function of $x$ inside the supremum on the right-hand side above is measurable. Indeed, we showed in Lemma 2.9 that $\mu \mapsto \rho_{\mu}(f)$ is measurable (actually lower semicontinuous) when $f$ is continuous and bounded. Since $C([0,1])$ equipped with the supremum norm is a Polish space, for a fixed $\epsilon>0$ we may find (by [9, Proposition 7.50]) a universally measurable map $E \ni x \mapsto g_{x} \in C([0,1])$ such that, for each $x \in E$,

$$
\int_{[0,1]} g_{x} d K_{x}^{v}-\rho_{K_{x}^{\mu}}\left(g_{x}\right) \geq \begin{cases}\alpha\left(K_{x}^{v} \mid K_{x}^{\mu}\right)-\epsilon & \text { if } \alpha\left(K_{x}^{v} \mid K_{x}^{\mu}\right)<\infty \\ 1 / \epsilon & \text { if } \alpha\left(K_{x}^{v} \mid K_{x}^{\mu}\right)=\infty .\end{cases}
$$

We may find a Borel measurable map which agrees $v$-a.e. with $x \mapsto g_{x}$, and we abuse notation by denoting this again by $x \mapsto g_{x}$. By Lusin's theorem, for each $\delta>0$ there exists a compact set $S_{\delta} \subset E$ such that $v\left(S_{\delta}^{c}\right) \leq \delta$ and the restriction $S_{\delta} \ni x \mapsto g_{x} \in C([0,1])$ is continuous. Without loss of generality, assume that $S_{\delta} \supset S_{\delta^{\prime}}$ whenever $\delta<\delta^{\prime}$. Define $g_{x}^{\delta}:=g_{x}$ for $x \in S_{\delta}$ and $g_{x}^{\delta}:=0$ (the zero function) for $x \notin S_{\delta}$, and note that $g^{\delta}$ is a bounded measurable function from $E$ to $C([0,1])$. Define $g^{\delta}: E \times[0,1] \rightarrow \mathbb{R}$ by $g^{\delta}(x, y)=g_{x}^{\delta}(y)$, and note that $\mathrm{g}^{\delta}$ is jointly measurable (thanks to Theorem 4.55 and Lemma 4.51 of [3]) and bounded.

Case 1. Suppose first that $\int_{E} v(d x) \alpha\left(K_{x}^{v} \mid K_{x}^{\mu}\right)<\infty$. Then, for sufficiently small $\delta$, we have

$$
\int_{S_{\delta}^{c}} v(d x) \alpha\left(K_{x}^{v} \mid K_{x}^{\mu}\right) \leq \epsilon .
$$

Then

$$
\begin{aligned}
\int_{E} v(d x) \alpha\left(K_{x}^{v} \mid K_{x}^{\mu}\right) & \leq \epsilon+\int_{S_{\delta}} v(d x) \alpha\left(K_{x}^{v} \mid K_{x}^{\mu}\right) \\
& \leq 2 \epsilon+\int_{S_{\delta}} v(d x)\left[\int_{[0,1]} g_{x} d K_{x}^{v}-\rho_{K_{x}^{\mu}}\left(g_{x}\right)\right] \\
& =2 \epsilon+\int_{E} v(d x)\left[\int_{[0,1]} g_{x}^{\delta} d K_{x}^{v}-\rho_{K_{x}^{\mu}}\left(g_{x}^{\delta}\right)\right] \\
& =2 \epsilon+\int_{E \times[0,1]} g^{\delta} d \bar{v}-\int_{E} v(d x) \rho_{K_{x}^{\mu}}\left(g^{\delta}(x, \cdot)\right) \\
& \leq 2 \epsilon+\sup _{f \in B(E \times[0,1])}\left\{\int_{E \times[0,1]} f d \bar{v}-\int_{E} v(d x) \rho_{K_{x}^{\mu}}(f(x, \cdot))\right\} .
\end{aligned}
$$

The second line used (A.2), and the third used the definition of $g^{\delta}$. Since $\epsilon>0$ was arbitrary, we obtain (A.1). 
Case 2. Suppose the set $I:=\left\{x \in E: \alpha\left(K_{x}^{v} \mid K_{x}^{\mu}\right)=\infty\right\}$ has $v(I)>0$. Then $\int_{E} v(d x) \alpha\left(K_{x}^{v} \mid K_{x}^{\mu}\right)=\infty$, and for small enough $\delta$ we have $v\left(S_{\delta} \cap I\right) \geq v(I) / 2>0$. Using (A.2) and the definition of $g^{\delta}$,

$$
\begin{aligned}
0<\frac{v(I)}{2 \epsilon} & \leq \frac{v\left(S_{\delta} \cap I\right)}{\epsilon}=\int_{S_{\delta} \cap I} \epsilon^{-1} v(d x) \\
& \leq \int_{S_{\delta} \cap I} v(d x)\left[\int_{[0,1]} g_{x} d K_{x}^{v}-\rho_{K_{x}^{\mu}}\left(g_{x}\right)\right] \\
& \leq \epsilon v\left(I^{c}\right)+\int_{E} v(d x)\left[\int_{[0,1]} g_{x}^{\delta} d K_{x}^{v}-\rho_{K_{x}^{\mu}}\left(g_{x}^{\delta}\right)\right] \\
& \leq \epsilon v\left(I^{c}\right)+\sup _{f \in B(E \times[0,1])}\left\{\int_{E \times[0,1]} f d \bar{v}-\int_{E} v(d x) \rho_{K_{x}^{\mu}}(f(x, \cdot))\right\} .
\end{aligned}
$$

The second to last inequality followed from the fact that

$$
\int_{I^{c}} v(d x) \int_{[0,1]} g_{x}^{\delta} d K_{x}^{v}-\rho_{K_{x}^{\mu}}\left(g_{x}^{\delta}\right) \geq-\epsilon v\left(I^{c}\right),
$$

by definition of $g^{\delta}$. Send $\epsilon \downarrow 0$ in (A.3) to conclude that the right-hand side of (A.1) is also infinite.

Case 3. Finally, suppose $\int_{E} v(d x) \alpha\left(K_{x}^{v} \mid K_{x}^{\mu}\right)=\infty$ but $v(I)=0$, with $I$ defined as in Case 2. Since $\alpha \geq 0$, the monotone convergence theorem implies

$$
\lim _{\delta \downarrow 0} \int_{S_{\delta}} v(d x) \alpha\left(K_{x}^{v} \mid K_{x}^{\mu}\right)=\infty .
$$

Fix $M>0$. Find $\delta$ such that $\int_{S_{\delta}} v(d x) \alpha\left(K_{x}^{v} \mid K_{x}^{\mu}\right) \geq M$. Then, again using (A.2), $v(I)=0$, and the definition of $g^{\delta}$,

$$
\begin{aligned}
M & \leq \epsilon+\int_{S_{\delta}} v(d x)\left[\int_{[0,1]} g_{x} d K_{x}^{v}-\rho_{K_{x}^{\mu}}\left(g_{x}\right)\right] \\
& =\epsilon+\int_{E} v(d x)\left[\int_{[0,1]} g_{x}^{\delta} d K_{x}^{v}-\rho_{K_{x}^{\mu}}\left(g_{x}^{\delta}\right)\right] \\
& \leq \epsilon+\sup _{f \in B(E \times[0,1])}\left\{\int_{E \times[0,1]} f d \bar{v}-\int_{E} v(d x) \rho_{K_{x}^{\mu}}(f(x, \cdot))\right\}
\end{aligned}
$$

Since $M>0$ was arbitrary, this shows that the right-hand side is infinite, verifying (A.1).

Proof for general $F$. Finally, we remove the assumption that $F=[0,1]$, merely assuming now that it is Polish. By Borel isomorphism (see [25, Theorem 15.6]) we may find a measurable bijection $T: F \rightarrow[0,1]$ with measurable inverse. Note that Proposition 2.6 yields $\alpha\left(K_{x}^{v} \mid K_{x}^{\mu}\right)=\alpha\left(K_{x}^{v} \circ T^{-1} \mid K_{x}^{\mu} \circ T^{-1}\right)$. Apply the above result 
to prove (A.1):

$$
\begin{aligned}
\int_{E} v(d x) \alpha\left(K_{x}^{v} \mid K_{x}^{\mu}\right) & =\int_{E} v(d x) \alpha\left(K_{x}^{v} \circ T^{-1} \mid K_{x}^{\mu} \circ T^{-1}\right) \\
& =\sup _{f \in B(E \times[0,1])} \int_{E} v(d x)\left[\int_{[0,1]} K_{x}^{v} \circ T^{-1}(d y) f(x, y)-\rho_{K_{x}^{\mu} \circ T^{-1}}(f(x, \cdot))\right] \\
& =\sup _{f \in B(E \times[0,1])} \int_{E} v(d x)\left[\int_{F} K_{x}^{v}(d y) f(x, T(y))-\rho_{K_{x}^{\mu}}(f(x, T(\cdot)))\right] \\
& \leq \sup _{f \in B(E \times F)} \int_{E} v(d x)\left[\int_{F} K_{x}^{v}(d y) f(x, y)-\rho_{K_{x}^{\mu}}(f(x, \cdot))\right] .
\end{aligned}
$$

Acknowledgement: Thanks are due to Igor Cialenco, Andrew Papanicolaou, and especially Stefan Weber for valuable discussions and references, as well as Kavita Ramanan for careful feedback on an early draft. Part of this research was performed while the author was visiting the Institute for Pure and Applied Mathematics (IPAM), which is supported by the National Science Foundation.

\section{References}

[1] Acciaio, B. and I. Penner (2011). Dynamic risk measures. In G. Di Nunno and B. Øksendal (Eds.), Advanced Mathematical Methods for Finance, pp. 1-34. Springer, Berlin.

[2] Acciaio, B. and G. Svindland (2013). Are law-invariant risk functions concave on distributions? Depend. Model. 1, 54-64.

[3] Aliprantis, C. D. and K. C. Border (2006). Infinite Dimensional Analysis: A Hitchhiker's Guide. Third edition. Springer, Berlin.

[4] Artzner, P., F. Delbaen, J.-M. Eber, and D. Heath (1999). Coherent measures of risk. Math. Finance 9(3), 203-228.

[5] Bartl, D. (2016). Conditional nonlinear expectations. Available at https://arxiv.org/abs/1612.09103v2.

[6] Bellini, F., V. Bignozzi, and G. Puccetti (2018). Conditional expectiles, time consistency and mixture convexity properties. Insurance Math. Econom. 82, 117-123.

[7] Ben-Tal, A. and M. Teboulle (1986). Expected utility, penalty functions, and duality in stochastic nonlinear programming. Manage. Sci. 32(11), 1445-1466.

[8] Ben-Tal, A. and M. Teboulle (2007). An old-new concept of convex risk measures: The optimized certainty equivalent. Math. Finance 17(3), 449-476.

[9] Bertsekas, D. P. and S. E. Shreve (1996). Stochastic Optimal Control: The Discrete Time Case. Athena Scientific, Belmont MA.

[10] Cheridito, P. and M. Kupper (2011). Composition of time-consistent dynamic monetary risk measures in discrete time. Int. J. Theor. Appl. Finance 14(01), 137-162.

[11] Csiszár, I. (2008). Axiomatic characterizations of information measures. Entropy 10(3), 261-273.

[12] Delbaen, F. (2002). Coherent Risk Measures on General Probability Spaces. In K. Sandmann and P. J. Schönbucher (Eds.), Advances in Finance and Stochastics, pp. 1-37. Springer, Berlin.

[13] Detlefsen, K. and G. Scandolo (2005). Conditional and dynamic convex risk measures. Finance Stoch. 9(4), 539-561.

[14] Eckstein, S. (2017). Extended Laplace principle for empirical measures of a Markov chain. Available at https://arxiv.org/abs/1709.02278.

[15] Filipović, D. and G. Svindland (2012). The canonical model space for law-invariant convex risk measures is $\underline{\text { is }} L^{1}$. Math. Finance 22(3), 585-589.

[16] Föllmer, H. and I. Penner (2006). Convex risk measures and the dynamics of their penalty functions. Stat. Decisions 24(1), 61-96.

[17] Föllmer, H. and A. Schied (2002). Convex measures of risk and trading constraints. Finance Stoch. 6(4), 429-447.

[18] Föllmer, H. and A. Schied (2011). Stochastic Finance: An Introduction in Discrete Time. De Gruyter, Berlin.

[19] Frittelli, M. and E. Rosazza Gianin (2002). Putting order in risk measures. J. Bank. Financ. 26(7), 1473-1486.

[20] Frittelli, M. and E. Rosazza Gianin (2004). Dynamic convex risk measures. In G. Szegö (Ed.), Risk Measures for the 21st Century, pp. 227-248. John Wiley \& Sons, Chichester.

[21] Frittelli, M. and E. Rosazza Gianin (2005). Law invariant convex risk measures. In S. Kusuoka and A. Yamazaki (Eds.), Advances in Mathematical Economics, pp. 33-46. Springer, Tokyo. 
[22] Heath, D. and H. Ku (2004). Pareto equilibria with coherent measures of risk. Math. Finance 14(2), 163-172.

[23] Jouini, E., W. Schachermayer, and N. Touzi (2006). Law invariant risk measures have the Fatou property. In S. Kusuoka and A. Yamazaki (Eds.), Advances in Mathematical Economics, pp. 49-71. Springer, Tokyo.

[24] Kallenberg, O. (2002). Foundations of Modern Probability. Second edition. Springer, New York.

[25] Kechris, A. S. (2012). Classical Descriptive Set Theory. Springer-Verlag, New York.

[26] Kullback, S. and R. Leibler (1951). On information and sufficiency. Ann. Math. Statist. 22(1), 79-86.

[27] Kupper, M. and W. Schachermayer (2009). Representation results for law invariant time consistent functions. Math. Financ. Econ. 2(3), 189-210.

[28] Kusuoka, S. (2001). On law invariant coherent risk measures. In S. Kusuoka and T. Maruyama (Eds.), Advances in Mathematical Economics, pp. 83-95. Springer, Tokyo.

[29] Lacker, D. (2016). A non-exponential extension of Sanov's theorem via convex duality. Available at https://arxiv.org/abs/1609.04744.

[30] Lacker, D. (2018). Liquidity, risk measures, and concentration of measure. Math. Oper. Res. 43(3), 693-1050.

[31] Liese, F. and I. Vajda (1987). Convex Statistical Distances. Teubner, Leipzig.

[32] Liese, F. and I. Vajda (2006). On divergences and informations in statistics and information theory. IEEE Trans. Inform. Theory 52(10), 4394-4412.

[33] Riedel, F. (2004). Dynamic coherent risk measures. Stochastic Process. Appl. 112(2), 185-200.

[34] Roorda, B. and J. M Schumacher (2007). Time consistency conditions for acceptability measures, with an application to Tail Value at Risk. Insurance Math. Econom. 40(2), 209-230.

[35] Shapiro, A. (2013). On Kusuoka representation of law invariant risk measures. Math. Oper. Res. 38(1), 142-152.

[36] Strassen, V. (1965). The existence of probability measures with given marginals. Ann. Math. Statist. 36(2), 423-439.

[37] Tutsch, S. (2008). Update rules for convex risk measures. Quantit. Finance 8(8), 833-843.

[38] Weber, S. (2006). Distribution-invariant risk measures, information, and dynamic consistency. Math. Finance 16(2), 419441.

[39] Winkler, G. (1985). Choquet Order and Simplices: With Applications in Probabilistic Models. Springer-Verlag, Berlin. 